\title{
Impact of non-equilibrium fluctuations on pre-thermal dynamical phase transitions in long-range interacting spin chains
}

\author{
Alessio Lerose \\ SISSA - International School for Advanced Studies, via Bonomea 265, I-34136 Trieste, Italy and \\ INFN - Istituto Nazionale di Fisica Nucleare, Sezione di Trieste, I-34136 Trieste, Italy \\ Bojan Žunkovič \\ Department of Physics, Faculty of Mathematics and Physics, \\ University of Ljubljana, Jadranska 19, 1000 Ljubljana, Slovenia \\ Jamir Marino \\ Department of Physics, Harvard University, Cambridge MA 02138, United States \\ Department of Quantum Matter Physics, University of Geneva, 1211, Geneve, Switzerland \\ Andrea Gambassi \\ SISSA - International School for Advanced Studies, via Bonomea 265, I-34136 Trieste, Italy \\ INFN - Istituto Nazionale di Fisica Nucleare, Sezione di Trieste, I-34136 Trieste, Italy \\ Alessandro Silva \\ SISSA - International School for Advanced Studies, via Bonomea 265, I-34136 Trieste, Italy
}

(Dated: March 5, 2019)

\begin{abstract}
We study the non-equilibrium phase diagram and the dynamical phase transitions occurring during the pre-thermalization of non-integrable quantum spin chains, subject to either quantum quenches or linear ramps of a relevant control parameter. We consider spin systems in which long-range ferromagnetic interactions compete with short-range, integrability-breaking terms. We capture the pre-thermal stages of the non-equilibrium evolution via a time-dependent spin-wave expansion at leading order in the spin waves density. In order to access regimes with strong integrability breaking, instead, we perform numerical simulations based on the time-dependent variational principle with matrix product states. By investigating a large class of quantum spin models, we demonstrate that non-equilibrium fluctuations can significantly affect the dynamics near critical points of the phase diagram, resulting in a chaotic evolution of the collective order parameter, akin to the dynamics of a classical particle in a multiple-well potential subject to quantum friction. We also elucidate the signature of this novel dynamical phase on the time-dependent correlation functions of the local order parameter. We finally establish a connection with the notion of dynamical quantum phase transition associated with a possible non-analytic behavior of the return probability amplitude, or Loschmidt echo, showing that the latter displays cusps whenever the order parameter vanishes during its real-time evolution.
\end{abstract}

\section{INTRODUCTION}

Consider an extended quantum many-body system in an equilibrium, low temperature, ordered phase (e.g. a ferromagnet), and drive it out of equilibrium by varying in time a control parameter (e.g., a magnetic field). This can occur via an abrupt change from an initial to a final value (the so-called quantum quench ${ }^{1-3}$ ), or a continuous time-dependent ramp. In these cases the initial long-range ordered state is destabilized, and it is therefore natural to investigate the fate of the order parameter out of equilibrium. If thermalization occurs quickly, the order parameter will show a behavior consistent with its equilibrium finite-temperature phase diagram. On the other hand, if a metastable, non-equilibrium quasisteady state is established at intermediate time scales before thermalization, non-trivial time-dependent phenomena may occur. This scenario, which focuses on prethermal states ${ }^{4-14}$, typically occurs in systems close to integrability. Quantum many body integrable systems are known to relax towards a generalized Gibbs ensemble (GGE), a sort of grand-canonical ensemble accounting for all the local (or quasi-local) conserved quantities of the system ${ }^{15-24}$. Under weak integrability-breaking perturbations, GGEs can act as metastable attractors of the dynamics, before the system slowly drifts towards its long-time, asymptotic steady state described by a canonical Gibbs ensemble ${ }^{25-34}$. In particular, the system lingers close to a state violating detailed balance in which conventional equilibrium statistical mechanics does not apply, making the onset of novel types of phases of matter and critical behavior possible.

An interesting example of non-equilibrium critical phenomena may emerge after a quantum quench of an interacting quantum many-body system which displays symmetry breaking at equilibrium. These dynamical phase transitions (DPTs) ${ }^{35-53}$ are characterized by a nonequilibrium order parameter exhibiting a finite or vanishing long-time temporal average, depending on whether 
the quantum model under consideration is quenched below or above an associated dynamical critical point separating the dynamical ordered phase from the dynamical disordered one which depends, inter alia, on the initial conditions. In addition, in systems with local interactions, the scaling of dynamical correlation and response functions can distinguish the different phases ${ }^{54-59}$. A recent experiment ${ }^{60}$ has shown that these dynamical phase transitions can be realized with ultracold trapped ions which simulate long-range interacting Ising ferromagnets. A second notion of DPT, proposed in Ref. 61, has been recently studied experimentally in Ref. 62. This notion, however, is not directly related to the existence of a local order parameter characterizing the various dynamical phases, but rather to a non-analytic behavior in the time dependence of the return probability amplitude. These two notions of dynamical phase transition are in general distinct, and therefore they may even not occur concomitantly in the same model. However, a connection has been pointed out whenever both DPTs are present ${ }^{63}$ (see also Refs. 64 and 65). The study of the two instances of DPTs is typically restricted to either integrable or mean-field models, or to numerical works, while Gaussian fluctuations have been accounted for in a limited number of cases ${ }^{45,46,49,56-59}$. The purpose of this paper is to thoroughly study DPTs in non-integrable models that, despite possessing non-trivial fluctuations and a non-vanishing order parameter, are amenable to an analytical approach.

As we anticipated in Ref. 66, the analysis of the fullyconnected quantum Ising model with an additional shortrange integrability-breaking perturbation reveals that fluctuations may induce unexpected "chaotic" behavior in the non-equilibrium dynamics: in the presence of $\mathbb{Z}_{2}$ symmetry breaking, the asymptotic sign of the order parameter turns out to depend sensitively, and to a large extent unpredictably, on the initial conditions and on the specific values of the parameters of the post-quench Hamiltonian. We thoroughly show here that this phenomenon turns out to rely on few essential physical ingredients, namely (i) the existence of multiple, macroscopically distinct equilibrium configurations for a collective order parameter, and (ii) the possibility of dissipating the energy of the collective motion into microscopic non-equilibrium fluctuations. In this work, these ingredients are provided by the competition between long- and short-range interactions in quantum many-body systems when quenched near a dynamical critical point: a mean-field collective degree of freedom moves in a multiple-well landscape and is weakly coupled to an extensive set of microscopic degrees of freedom which represent quantum fluctuations at all length scales. The latter provide a sort of quantum friction on the classical collective motion that in turn makes the eventual "choice" of the asymptotic well highly sensitive to the parameters. This behavior is actually reminiscent of what is observed in a coin toss ${ }^{67}$, with the coin playing the role of the macroscopic collective degree of freedom with two possible stable equilibrium configurations ("heads" and "tails"), which dissipates its energy into the microscopic degrees of freedom (phonons of the floor, air molecules,...) and finally undergoes a pseudo-random choice of the asymptotic state. The peculiar behavior described above is based on general properties and is therefore expected to occur in a variety of systems. Here we will first illustrate it in the case of the fully-connected quantum Ising model in a transverse field, i.e., the Lipkin-Meshkov-Glick (LMG) model, and later on we will discuss a much more general class of models where the phenomenon is observed. Moreover, we investigate the signatures of this phenomenon in the non-equilibrium spreading of correlations, as well as its occurrence with more general non-equilibrium protocols such as linear ramps.

The paper is organized as follows. In Sec. II we review the properties of both the equilibrium and the dynamical criticality of the LMG model which represents the basis for the analysis presented in the following Sections.

Sec. III illustrates in a pedagogical fashion the methods used in this study, based on a spin wave (HolsteinPrimakoff) expansion around the instantaneous average direction of the spins, whose evolution is self-consistently determined by taking into account the feedback from the quantum spin wave fluctuations. This approach is suitable for studying both equilibrium and non-equilibrium problems in a wide range of systems close to mean-field integrability (i.e., long-range or high-dimensional systems), and is therefore of interest by itself.

In Secs. IV A and IV B we discuss in detail the impact of integrability-breaking perturbations on the dynamical phase diagram of mean-field models, showing explicitly, through an extensive analysis encompassing several different types of perturbations and generalizations of the LMG model, that the chaotic dynamical phase found in Ref. 66 has to be expected in general.

Sec. IV C is devoted to the calculation of the equaltime correlation function of the order parameter at different space points across the dynamical phase diagram of the LMG model perturbed by nearest-neighbor transverse spin interactions. These correlation functions exhibit a periodic modulation in time, illustrating that the dynamics of the spin waves is periodically self-driven as a result of the precession of the collective magnetization of the LMG model induced by the transverse field.

Finally, in Sec. IVD results are presented for a linear ramp of the transverse field as a function of time in the LMG model, generalizing the sudden quench considered in Sec. IV. As the duration of the ramp increases, the chaotic phase shrinks in the adiabatic limit; on the contrary, the faster the ramp is, the closer the dynamical phase diagram is to the one generated by a sudden quench (Sec. IV).

In Sec. V A we confirm the onset of the chaotic phase for strong integrability-breaking perturbations by employing a numerical method based on matrix product states, extending the findings of Ref. 66 .

In Sec. VB we discuss the connection between the 
dynamical phase transition discussed in this paper and the notion of dynamical phase transition associated with cusps of the Loschmidt echo ${ }^{61}$, confirming also in the present case the prediction of Ref. 63: whenever the order parameter vanishes during its evolution, cusps are concomitantly formed in the real-time dynamics of the return probability amplitude.

In Sec. VI we discuss the important issue of finite-size effects, relevant to possible experimental realizations of the phenomena hereby discussed.

In Sec. VII we discuss further perspectives.

\section{DYNAMICAL PHASE TRANSITION IN THE INFINITE-RANGE ISING MODEL}

In this work we firstly focus on the non-equilibrium dynamics of a general class of Ising-type systems with quantum $s$-spins on a lattice, interacting via ferromagnetic coupling and subject to a transverse magnetic field

$$
H=-\sum_{\mathbf{r}, \mathbf{r}^{\prime}} J_{\left|\mathbf{r}-\mathbf{r}^{\prime}\right|} \sigma_{\mathbf{r}}^{x} \sigma_{\mathbf{r}^{\prime}}^{x}-g \sum_{\mathbf{r}} \sigma_{\mathbf{r}}^{z}
$$

where the sums run over the sites of a lattice, while $\sigma_{\mathbf{r}}^{\alpha}=S_{\mathbf{r}}^{\alpha} / s$ are the operators corresponding to the normalized spin components in the $\alpha=x, y, z$ direction, acting on site $\mathbf{r}$. This represents a generalization of the case of spins one-half, where $s=1 / 2$ and the $\sigma_{\mathbf{r}}^{\alpha}$ 's reduce to the standard Pauli matrices. Controlling $s$ allows us to keep track of the impact of quantum fluctuations, which is suppressed in the classical limit $s \rightarrow \infty$. The ferromagnetic couplings $J_{r}$ depend on the distance $r=\left|\mathbf{r}-\mathbf{r}^{\prime}\right|$ between two sites.

For general ferromagnetic interactions $J_{r}$ (short- or long-range), the system is expected to have an equilibrium zero-temperature phase transition from a unique paramagnetic ground state with $\left\langle\sigma^{x}\right\rangle=0$ for $g>g_{\text {cr }}$ to a pair of ferromagnetic ground states with $\left\langle\sigma^{x}\right\rangle_{ \pm}=$ $\pm m \neq 0$ for $g<g_{\text {cr }}$, characterized by the breaking of the $\mathbb{Z}_{2}$-symmetry $\sigma^{x} \mapsto-\sigma^{x}$. The emergence of a non-vanishing order parameter at a finite energy density above the ground state (e.g., in an equilibrium finitetemperature state, or in a non-equilibrium state attained after a quench) depends on the dimensionality and on the range of the interactions. While one-dimensional systems with short-range interactions cannot support order in excited states ${ }^{68,69}$, this is possible in models with either higher spatial dimensionality or with long-range interactions. In these cases, a non-vanishing order parameter may persist in thermal as well as in pre-thermal phases.

The simplest instance of the generic Hamiltonian (1) is that with $J_{r}=\lambda / N$, corresponding to the infinite-range or fully-connected model ${ }^{70,71}$

$$
H=-\frac{\lambda}{N} \sum_{i, j=1}^{N} \sigma_{i}^{x} \sigma_{j}^{x}-g \sum_{i=1}^{N} \sigma_{i}^{z},
$$

where each of the $N$ spins interacts with all the others with the same ferromagnetic coupling strength, $\lambda / N$. This is equivalent to the Lipkin-Meshkov-Glick model ${ }^{70}$. The rest of Sec. II is devoted to reviewing the equilibrium and non-equilibrium behavior of this paradigmatic model, focusing on dynamical phase transitions after a quench. The readers familiar with this may skip to Sec. III, in which we discuss fluctuations in the presence of perturbations.

The $1 / N$ scaling of $J_{r}$ in Eq. (2) is necessary in order to make the energy extensive in the thermodynamic limit. As $N \rightarrow \infty$ the mean-field approximation becomes exact for the Hamiltonian (2), and therefore the model is exactly solvable in the thermodynamic limit. Indeed, $H$ is solely a function of the total spin components

$$
H=-\frac{\lambda}{N}\left(\tilde{\sigma}_{k=0}^{x}\right)^{2}-g \tilde{\sigma}_{k=0}^{z},
$$

where $\tilde{\sigma}_{k=0}^{\alpha}=\sum_{i} \sigma_{i}^{\alpha}$ is the Fourier mode with zero momentum $k=0$ of the spins on the lattice. All the other degrees of freedom $\tilde{\sigma}_{k \neq 0}^{\alpha}$, corresponding to the spatial fluctuations with $k \neq 0$ in Fourier space of the spins, do not contribute to the dynamical properties of the model (3).

The Hamiltonian $H$ is diagonalizable separately in each sector of fixed total spin magnitude $(N s-m)(N s-$ $m+1$ ), with $m=0,1, \ldots, N s$ or $N s-1 / 2$ (depending on $N s$ being integer or half integer respectively). When $N \rightarrow \infty$, these sectors can be labelled by a continuous variable,

$$
\left|\tilde{\vec{\sigma}}_{k=0}\right| / N \rightarrow \rho
$$

with $0 \leq \rho \leq 1$. The ground state always belongs to the maximal total spin sector, $\left|\tilde{\vec{\sigma}}_{k=0}\right|=N$ or $\rho=1$. Accordingly, this state has extensive quantum numbers, and the thermodynamic limit $N \rightarrow \infty$ is equivalent to the semiclassical limit, or, in loose terms, to a classical, continuous spin $\vec{\sigma} \equiv\left\langle\tilde{\vec{\sigma}}_{k=0}\right\rangle / N$ of (conserved) length $\rho$. The behavior of the system in that limit is then completely determined by the classical Hamiltonian

$$
\mathcal{H}_{\mathrm{cl}}(\vec{\sigma})=-\lambda\left(\sigma^{x}\right)^{2}-g \sigma^{z},
$$

corresponding to the quantity $H / N$, where $\vec{\sigma}$ is now a classical spin, its phase space being the surface of a sphere of radius $0<\rho \leq 1$. The rigorous version of this statement is the following: When $N \rightarrow \infty$, the ground state expectation value of the spins $\left\langle\vec{\sigma}_{i}\right\rangle$ is given by the minimum point of $\mathcal{H}_{\mathrm{cl}}$ on the sphere, while its non-equilibrium evolution $\left\langle\vec{\sigma}_{i}(t)\right\rangle$ with a possibly time-dependent field $g(t)$, starting from a fully polarized state, is given by the corresponding classical trajectory on the sphere governed by $\mathcal{H}_{\mathrm{cl}}$ via the equations of motion $\dot{\sigma}^{\alpha}=\left\{\sigma^{\alpha}, \mathcal{H}_{\mathrm{cl}}\right\}$, with $\left\{\sigma^{\alpha}, \sigma^{\beta}\right\}=\varepsilon^{\alpha \beta \gamma} \sigma^{\gamma}$ and with time rescaled by $s$.

\section{A. Equilibrium behavior}

For a given sphere radius $\rho$, the classical Hamiltonian (5) has a single minimum for large $g$ with $\sigma^{x}=$ 


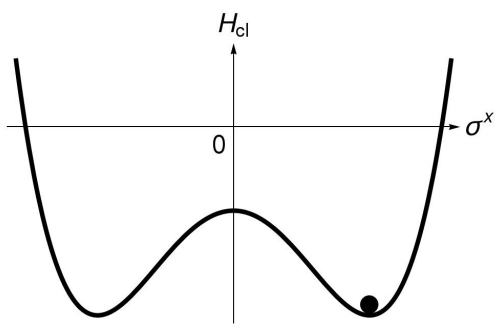

Figure 1. Classical energy landscape (5) of the collective spin $\vec{\sigma}$ of the LMG model along the plane $\sigma^{y} \equiv 0$ as a function of the magnetization $\sigma^{x}$, in the ferromagnetic phase $0<g<g_{\text {cr }} \equiv 2 \lambda \rho$. The location of the two symmetric minima is determined by Eq. (7). In the thermodynamic limit, the degenerate ground state wavefunctions of the collective spin are localized at the two classical minima respectively, and $\vec{\sigma}$ behaves like a classical particle at rest at the bottom of one of the two wells (e.g., black dot in the figure). At finite size, however, quantum tunneling induced by the presence of the other well occurs over an exponentially long time scale, see Sec. II C.
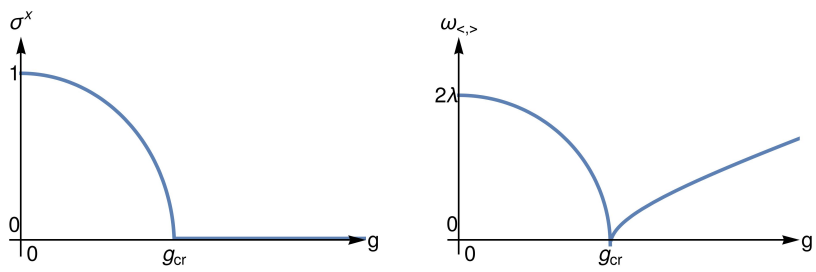

Figure 2. Left panel: Equilibrium order parameter $\sigma^{x}$ of the infinite-range Ising model at zero-temperature as a function of the external field $g$, determined by Eq. (7). Right panel: Frequency $\omega_{<,>}$of small oscillations of the collective spin around the minimum, see Eqs. (11) and (14), equal to the energy gap above the ground state. In both cases, the critical behavior is characterized by a square root singularity.

$\sigma^{y}=0, \sigma^{z}=\rho$, corresponding to a paramagnetic phase. As the strength of the field decreases below the critical value

$$
g_{\text {cr }} \equiv 2 \lambda \rho,
$$

that minimum bifurcates into a pair of minima characterized by non-vanishing, opposite magnetizations $\sigma^{x}$ along the $x$-direction, located on the $x z$-plane symmetrically with respect to the inversion of the $x$-axis [i.e., connected by the $\mathbb{Z}_{2}$ symmetry of the Hamiltonian (2)]. The corresponding double-well energy landscape is represented in Fig. 1. Parameterizing $\vec{\sigma}$ with spherical angles $(\theta, \phi)$, i.e., as $\vec{\sigma}=\rho(\sin \theta \cos \phi, \sin \theta \sin \phi, \cos \theta)$, the two ferromagnetic minima are given by $\left(\theta^{*}, 0\right)$ and $\left(\theta^{*}, \pi\right)$, with

$$
\cos \theta^{*}=\frac{g}{g_{\mathrm{cr}}} .
$$

Accordingly, the value of order parameter is

$$
\sigma^{x}= \pm \rho \sin \theta^{*}= \pm \rho \sqrt{1-\left(g / g_{\mathrm{cr}}\right)^{2}}
$$

(see the left panel of Fig. 2).
Let us now determine the spectrum of the lowest excitations above the ground states discussed above. Within each sector with fixed value of the total spin magnitude, labelled by $\rho$, the quantum mechanics of the collective spin is equivalent to that of a quantum particle in a potential well whose depth grows proportionally to $N$. (The absolute ground state sector corresponds to $\rho=1$.) In the thermodynamic limit, the lowest excitations of this particle are harmonic, and are determined by the quadratic expansion of the Hamiltonian around its energy minimum(a). This can be seen by a simple HolsteinPrimakoff transformation, as we discuss below.

For $g>g_{\text {cr }}$ the minimum occurs at $\theta=0$, and in terms of tangent canonical coordinates $q, p$, with $[q, p]=$ $i$, the quantum fluctuations around that minimum take the form

$$
\begin{aligned}
& s \tilde{\sigma}_{k=0}^{z}=N s \rho-n=N s \rho-\frac{q^{2}+p^{2}-1}{2}, \\
& s \tilde{\sigma}_{k=0}^{x} \approx \sqrt{N s \rho} q, \\
& s \tilde{\sigma}_{k=0}^{y} \approx \sqrt{N s \rho} p .
\end{aligned}
$$

The quantum number $n=0,1,2, \ldots$ labels the quantized spin projection along the direction of the minimum. The Hamiltonian (3) becomes, using Eqs. (9),

$$
\begin{aligned}
H_{>} & =-N g \rho+\frac{g}{s} \frac{q^{2}+p^{2}-1}{2}-\frac{\lambda \rho}{s} q^{2}= \\
& =-N g \rho+\frac{1}{s}\left(\frac{\omega_{>}-\omega_{>}^{(0)}}{2}\right)+\frac{1}{s} \omega_{>} n,
\end{aligned}
$$

where

$$
\omega_{>}=\sqrt{g\left(g-g_{\mathrm{cr}}\right)}, \quad \omega_{>}^{(0)}=g .
$$

The first term in the last line of Eq. (10) represents the classical energy [compare with Eq. (5)], the second one is the quantum zero-point energy contribution, i.e., the energy increase due to the quantum fluctuations of the spin around the classical minimum configuration, while the last one is the energy of the elementary (harmonic) excitations, with $n=0,1,2, \ldots$.

For $g<g_{\mathrm{cr}}$, the two minima of the classical Hamiltonian are determined by Eq. (7). Introducing the canonical coordinates given by the total spin projection $P$ along $z$ and the conjugated angle $Q$,

$$
\begin{aligned}
& P=s \tilde{\sigma}_{k=0}^{z}=N s \rho \cos \theta, \\
& Q=\phi
\end{aligned}
$$

respectively, and expanding the Hamiltonian (3) around one of the two classical minima $\left(\theta^{*}, 0\right)$ or $\left(\theta^{*}, \pi\right)$ with $\theta^{*}$ given by Eq. (7) (by symmetry the excitations spectra are identical)

$$
P=N s \rho \cos \theta^{*}+\delta P, \quad Q=\phi^{*}+\delta Q,
$$


we get

$$
\begin{aligned}
H_{<}= & -N\left(g \rho \cos \theta^{*}+\lambda \rho^{2} \sin ^{2} \theta^{*}\right) \\
& -\frac{1}{2 s}\left(g \cos \theta^{*}+2 \lambda \rho \sin ^{2} \theta^{*}\right) \\
& +\frac{2 \lambda}{s}\left[\frac{1}{N s} \frac{(\delta P)^{2}}{2}+N \rho^{2} \sin ^{2} \theta^{*} \frac{(\delta Q)^{2}}{2}\right] \\
= & -N\left(\frac{g^{2}}{4 \lambda}+\lambda \rho^{2}\right)+\frac{1}{s}\left(\frac{\omega_{<}-\omega_{<}^{(0)}}{2}\right)+\frac{1}{s} \omega_{<} n,
\end{aligned}
$$

where

$$
\omega_{<}=\sqrt{g_{\mathrm{cr}}^{2}-g^{2}}, \quad \omega_{<}^{(0)}=g_{\mathrm{cr}} .
$$

Analogously to Eq. (10), the first term in the last line of Eq. (13) represents the classical energy [compare with Eq. (5)], the second one is the quantum zero-point energy contribution, i.e., the energy increase due to the quantum fluctuations of the spin around the classical minimum configuration, while the last one is the energy of the elementary (harmonic) excitations, with $n=0,1,2, \ldots$. We observe that the energy gap above the ground state closes at the equilibrium critical point $g=g_{\mathrm{cr}}$, with a mean-field critical exponent $1 / 2$ (see Fig. 2).

In principle, one could think of including modes at finite $k \neq 0$ (spin waves) which would however, in this limit, be decoupled from the dynamics of the zero-mode. If $N_{\mathrm{sw}}=0,1,2, \ldots$ is the total occupation number of the spin wave modes with $k \neq 0$, the collective spin magnitude is $\left(N s-N_{\mathrm{sw}}\right)\left(N s-N_{\mathrm{sw}}+1\right)$, i.e.,

$$
\rho=\frac{\left|\tilde{\vec{\sigma}}_{k=0}\right|}{N}=1-\frac{N_{\mathrm{sw}}}{N s} .
$$

Hence one finally obtains from Eqs. (11) and (14) the complete spectrum of excitations above the ground state, in the thermodynamic limit and to leading order in $N_{\mathrm{sw}}$,

$$
\begin{aligned}
& H_{>}=-N g+\frac{\omega_{>}-\omega_{>}^{(0)}}{2 s}+\frac{1}{s}\left(g N_{\mathrm{sw}}+\omega_{>} n\right), \\
& H_{<}=-N\left(\frac{g^{2}}{4 \lambda}+\lambda\right)+\frac{\omega_{<}-\omega_{<}^{(0)}}{2 s}+\frac{1}{s}\left(2 \lambda N_{\mathrm{sw}}+\omega_{<} n\right),
\end{aligned}
$$

valid for $g>2 \lambda$ and $g<2 \lambda$, respectively. All the spin wave excitations introduced above have finite gap $g / s$ or $2 \lambda / s$ and a flat dispersion relation independent of the wavevector $k \neq 0$, because the fully-connected interactions carry no information on spatial scales, hence cannot resolve finite wavelengths. As a consequence, the presence of a finite low temperature $T$ leads to exponentially small corrections to the order parameter, with a shift of the critical point that can be computed ${ }^{71}$ by minimizing the mean-field classical Hamiltonian (5) with

$$
\rho(T)=1-\frac{1}{s} \frac{1}{e^{\frac{2 \lambda / s}{T}}-1} .
$$

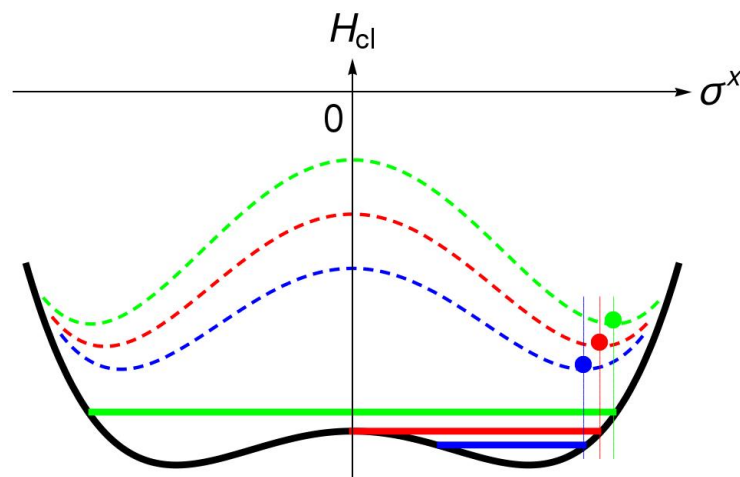

Figure 3. [Color online] Classical energy landscapes (5) of the collective spin $\vec{\sigma}$ of the LMG model in the plane $\sigma^{y} \equiv 0$ as a function of the magnetization $\sigma^{x}$ in the ferromagnetic phase, with a post-quench value $g$ such that $0<g<g_{\text {cr }}$ (black solid line) and several possible pre-quench values $g_{0}$ such that $0<g_{0}<g$ (blue, red and green dashed lines) of the transverse magnetic field. If the system is prepared in a ground state, e.g., with positive magnetization as illustrated by the blue, red, and green dots for decreasing values of $g_{0}$, and the magnetic field is suddenly quenched to a larger value $g_{0}<g<g_{\mathrm{cr}}$, then depending on the strength $g-g_{0}$ of the quench, the resulting non-equilibrium evolution may display dynamical ferromagnetic or paramagnetic behavior, exemplified by the blue and green line, respectively, separated by a critical trajectory with a diverging period, corresponding to the red line and associated with the dynamical critical point $g=g_{\text {dyn }}$. In contrast to Fig. 4 , here the various resulting evolutions correspond to varying the pre-quench parameter $g_{0}$, with a fixed post-quench value $g$.

\section{B. Dynamical criticality}

After setting the stage, let us now focus on the dynamics of interest in this work. Suppose that the system is prepared at time $t=0$ in a ferromagnetic ground state of the Hamiltonian (2) with a transverse field $g_{0}<g_{\mathrm{cr}}=2 \lambda$. Microscopically, this state is close to a spin-coherent state with all the spins aligned in the direction $\left(\theta^{*}, 0\right)$ or $\left(\theta^{*}, \pi\right)$ with $\cos \theta^{*}=g / g_{\mathrm{cr}}$, see Eq. (7), and with subextensive zero-point fluctuations of the collective spin, see Eq. (16). Then, the external field is suddenly increased to $g>g_{0}$, faster than the typical timescale of the system's dynamics. As argued above, the spins will initiate a collective precession, and the evolution of their direction on the sphere will be described by the classical trajectory of the post-quench Hamiltonian $\mathcal{H}_{\mathrm{cl}}(g)$, with the initial data corresponding to the minimum of the pre-quench Hamiltonian $\mathcal{H}_{\mathrm{cl}}\left(g_{0}\right)$, see Eq. (5).

Depending on the strength $g-g_{0}$ of the quench $g_{0} \rightarrow g$ of the transverse field, starting from a ferromagnetic pre-quench Hamiltonian, the resulting dynamics display qualitatively different orbits $^{42,72}$, as shown in Figs. 3 and 4:

1. For a shallow quench $\left[g<g_{\text {dyn }} \equiv\left(g_{0}+g_{\text {cr }}\right) / 2\right]$, the post-quench energy remains below the top of 

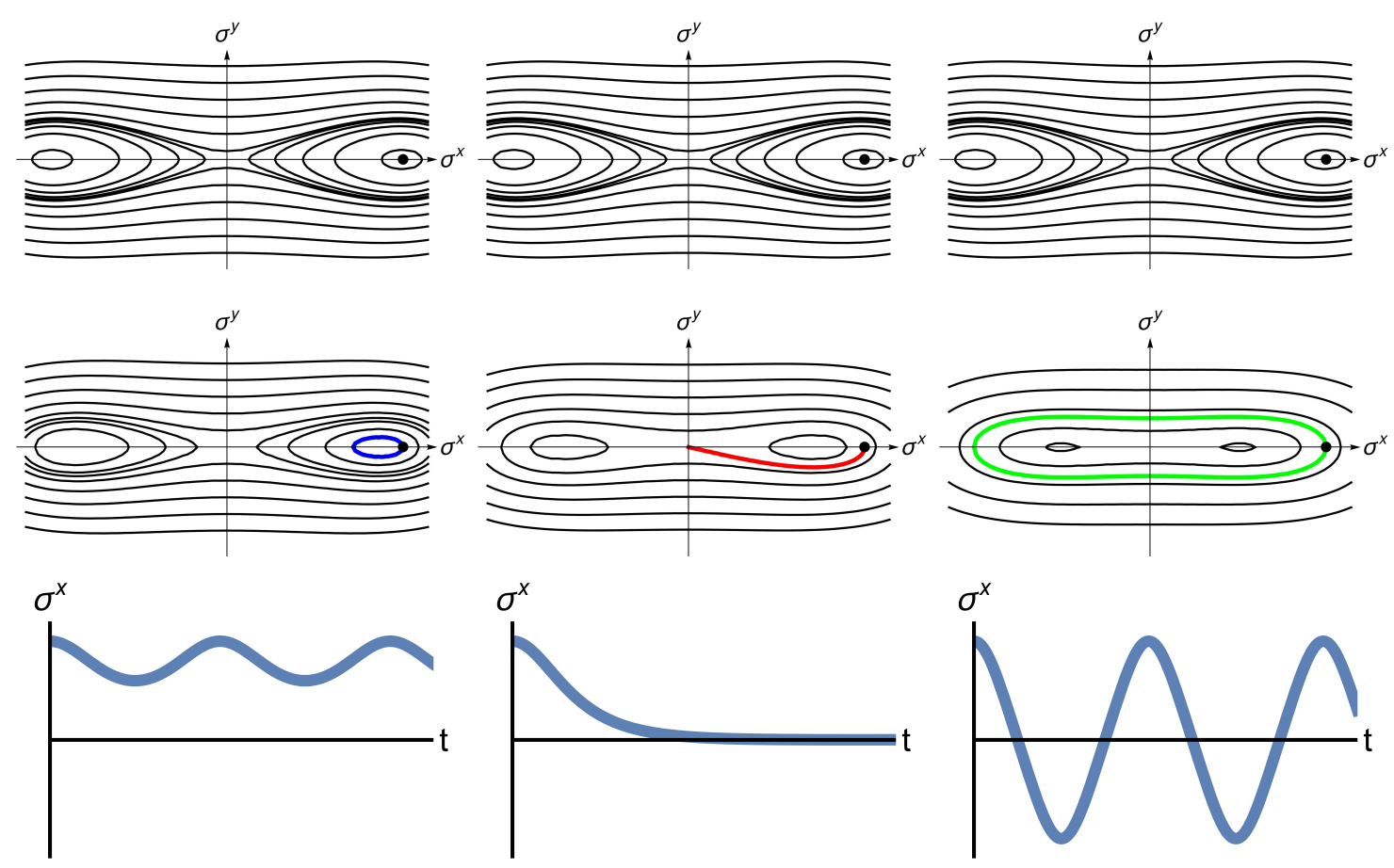

Figure 4. [Color online] Non-equilibrium dynamics of the LMG model (2) in the thermodynamic limit, after a sudden quench $g_{0} \rightarrow g$ of the transverse magnetic field starting from a ferromagnetic ground state of $H\left(g_{0}\right)$. The first row shows the semiclassical phase portrait of the pre-quench Hamiltonian $\mathcal{H}_{\mathrm{cl}}\left(g_{0}\right)$, where the initial state is represented by one of the two minima. The second row shows the semiclassical phase portrait of the post-quench Hamiltonian $\mathcal{H}_{\mathrm{cl}}(g)$, where the initial state is no longer a stationary point but moves along a non-trivial non-equilibrium trajectory, in the three qualitatively different cases corresponding to $g<g_{\mathrm{dyn}}, g=g_{\mathrm{dyn}}$ and $g>g_{\mathrm{dyn}}$ in the first, second and third column, respectively. The third row shows the dynamics of the order parameter as a function of time for the three cases. First column: for a weak quench, the dynamics remain trapped within the starting ferromagnetic sector; second column: for the critical quench, the initial state lies on a separatrix of the post-quench Hamiltonian and its subsequent evolution approaches the unstable equilibrium point at infinite time; third column: for a strong quench, the semiclassical orbit encircles both ferromagnetic minima, hence the symmetry is dynamically restored and the time-averaged order parameter is zero. In contrast to Fig. 3, here the different trajectories correspond to a varying post-quench parameter $g$, with a fixed pre-quench value $g_{0}$.

the barrier that separates the two ferromagnetic sectors. Correspondingly, the spin will precess within the starting ferromagnetic sector (blue lines in Figs. 3 and 4).

2. As the strength of the quench increases, the precession period $T_{\mathrm{cl}}=2 \pi / \Omega_{\mathrm{cl}}$ (which depends on both $g_{0}$ and $g$ ) increases, until for $g \nearrow g_{\mathrm{dyn}}$ it takes an infinite time to complete one cycle, and the unstable point at the top of the energy barrier is approached exponentially fast along the classical separatrix (red lines in Figs. 3 and 4).

3. For deep quenches above this threshold $g>g_{\mathrm{dyn}}$, the corresponding post-quench energy is larger than the barrier and the orbit of the collective spin on the sphere encircles both minima, such that the symmetry is dynamically restored after taking time-averages.
In fact, the time-average

$$
\overline{\sigma^{x}}=\lim _{T \rightarrow \infty} \frac{1}{T} \int_{0}^{T} d t \sigma^{x}(t)
$$

of the equilibrium order parameter $\sigma^{x}$ as a function of the quench strength, vanishes abruptly at the dynamical critical value $g_{\mathrm{dyn}}$ of the transverse field which depends also on the initial condition. This dynamical critical point separates a dynamical ferromagnetic phase with $\overline{\sigma^{x}} \neq 0$ from a dynamical paramagnetic phase with $\overline{\sigma^{x}}=0$.

The vanishing of an order parameter and the divergence of a characteristic time scale such as those reported in Fig. 5 are usually associated with critical phenomena. However, the system under consideration is clearly out of thermal equilibrium, as all microscopic spins perform a coherent, undamped precession. For this reason the above phenomenology can be described as dynamical criticality. In order to reinforce the idea that this behavior is distinct from the corresponding equilibrium phase transition, we emphasize that the equilibrium singularity of the order parameter upon approaching a critical 

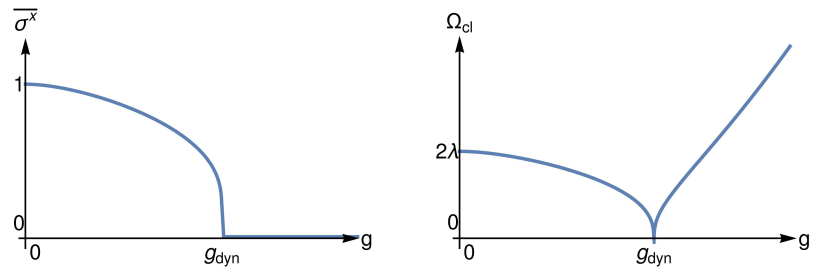

Figure 5. Left panel: Non-equilibrium order parameter $\overline{\sigma^{x}}$, defined in Eq. (18), of the infinite-range Ising model (2) after a quench of the external magnetic field starting from a ferromagnetic ground state with $g_{0}=0$ and positive magnetization, as a function of the post-quench field $g$. Right panel: Classical frequency $\Omega_{\mathrm{cl}}$ of the mean-field dynamical trajectory, which represents the characteristic time scale of the nonequilibrium evolution, as a function of the post-quench field $g$. For both quantities, the nature of the singular behavior at the dynamical critical point $g=g_{\text {dyn }}$ is logarithmic, as explained in the text. These plots can be compared with the analogous ones in equilibrium conditions in Fig. 2.

point has a critical exponent $1 / 2$, see Fig. 2, whereas the non-equilibrium order parameter $\overline{\sigma^{x}}$ actually displays a logarithmic singularity. Indeed, the divergence of the period of the classical oscillations as $g \nearrow g_{\mathrm{dyn}}$ is of the same form as that of a classical pendulum as the initial position approaches the upper configuration, with vanishing initial velocity ${ }^{73}$, and therefore the time average $\overline{\sigma^{x}}$ inherits the same type of singularity.

The dynamical criticality thoroughly discussed here is not peculiar of the Ising ferromagnets or of sudden quenches. Rather, it is a general feature of mean-field models driven away from equilibrium ${ }^{42,43}$. If the driving is chosen to be a slow ramp of the value of $g$ instead of a sudden quench, the dynamical critical point retains its nature, although it gets shifted towards the equilibrium critical point, until it merges with the latter in the limit of adiabatic variation.

\section{Finite-size (quantum) corrections}

In order to understand the possible connection with experimental realizations of long-range models, we now discuss the quantum corrections to the above classical behavior, which are relevant when the size $N$ of the system is finite. ${ }^{74}$

As we have argued above, the infinite-range Hamiltonian (2) describes the dynamics of the a single collective degree of freedom, namely $\vec{\sigma} \equiv \sum_{i=1}^{N} \vec{\sigma}_{i} / N$. In fact, the operators $\sigma^{\alpha}$, with $\alpha=x, y, z$, have spectrum in $[-1,1]$ and satisfy

$$
\left[\sigma^{\alpha}, \sigma^{\beta}\right]=\frac{1}{N s} i \epsilon^{\alpha \beta \gamma} \sigma^{\gamma},
$$

which implies that an effective Planck's constant $\hbar_{\mathrm{eff}} \equiv$ $1 /(N s)$ characterizes the quantum dynamics ${ }^{42}$. For this reason, the corrections to the classical motion can be in- vestigated via a semiclassical expansion in inverse powers of $N$ of the solution of the Schrödinger equation.

Let us now discuss the non-equilibrium dynamics within the semiclassical approximation ${ }^{75,76}$. The first quantum correction to the classical evolution starting from a spin-coherent state is equivalent to treating the corresponding Gaussian Wigner function in phase space as a probability distribution and considering its classical (Liouville) evolution. To this level of approximation, known as the truncated Wigner approximation (TWA), the role of quantum mechanics amounts just to providing a degree of uncertainty to the classical phase space point which represents the initial state of the system ${ }^{77}$. The amount of uncertainty is quantified by the phase space extension of the wavepacket, which covers an area equal to Planck's constant $h$, corresponding to the maximal phase space resolution allowed by the Heisenberg uncertainty relation.

In the presence of a non-quadratic Hamiltonian, like the one in Eq. (5) in which we are interested in this work, closeby points in phase space separate linearly in time, due to their different periods, with the sole exception of the critical trajectory with diverging period, around which two points separate exponentially fast in time ${ }^{78}$. Accordingly, since the linear extension of the initial wavepacket in phase space is $\sqrt{\hbar_{\mathrm{eff}}} \sim$ $1 / \sqrt{N}$, after a timescale of order $\mathcal{O}(\sqrt{N})[\operatorname{or} \mathcal{O}(\log \sqrt{N})$ around the separatrix] (the so-called Ehrenfest time $t_{\mathrm{Eh}}$ ) the wavepacket spreads over the whole classical trajectory, and the observables relax to their "microcanonical" average ${ }^{42,75,76,79}$.

In the light of the above, the qualitative modifications of the classical dynamics discussed in the previous section due to finite-size effects can be summarized as follows:

1. a fully polarized spin-coherent initial state actually corresponds to a broad wavepacket of linear extension $\propto 1 / \sqrt{N}$ on the sphere of radius 1 , rather than to a single point in phase space;

2 . in order to observe the classical evolution described in the previous section, the thermodynamic limit must be taken first: at finite $N$, instead, quantummechanical effects such as the wavepacket spreading set in after the time scale $T_{\text {Eh }} \sim \mathcal{O}(\sqrt{N})$ [or $\mathcal{O}(\log N)$ around the dynamical critical point] and the persistent classical oscillations are correspondingly damped;

3. the sharp dynamical phase transition highlighted in the previous section is smoothed out by quantum fluctuations, resulting in a crossover.

The four relevant time scales $T_{\mathrm{cl}}=\mathcal{O}(1)$ (classical period), $T_{\mathrm{Eh}}=\mathcal{O}(\sqrt{N})$ (wavepacket-spreading time scale), $T_{\text {rec }}=\mathcal{O}(N)$ (wavepacket recurrence time), $T_{\text {tun }}=$ $\mathcal{O}\left(e^{c N}\right)$ (tunneling time), are all well separated in the thermodynamic limit. Despite all quantum phenomena set in at increasingly longer time with $N$, in small systems they become important. In order to highlight the 

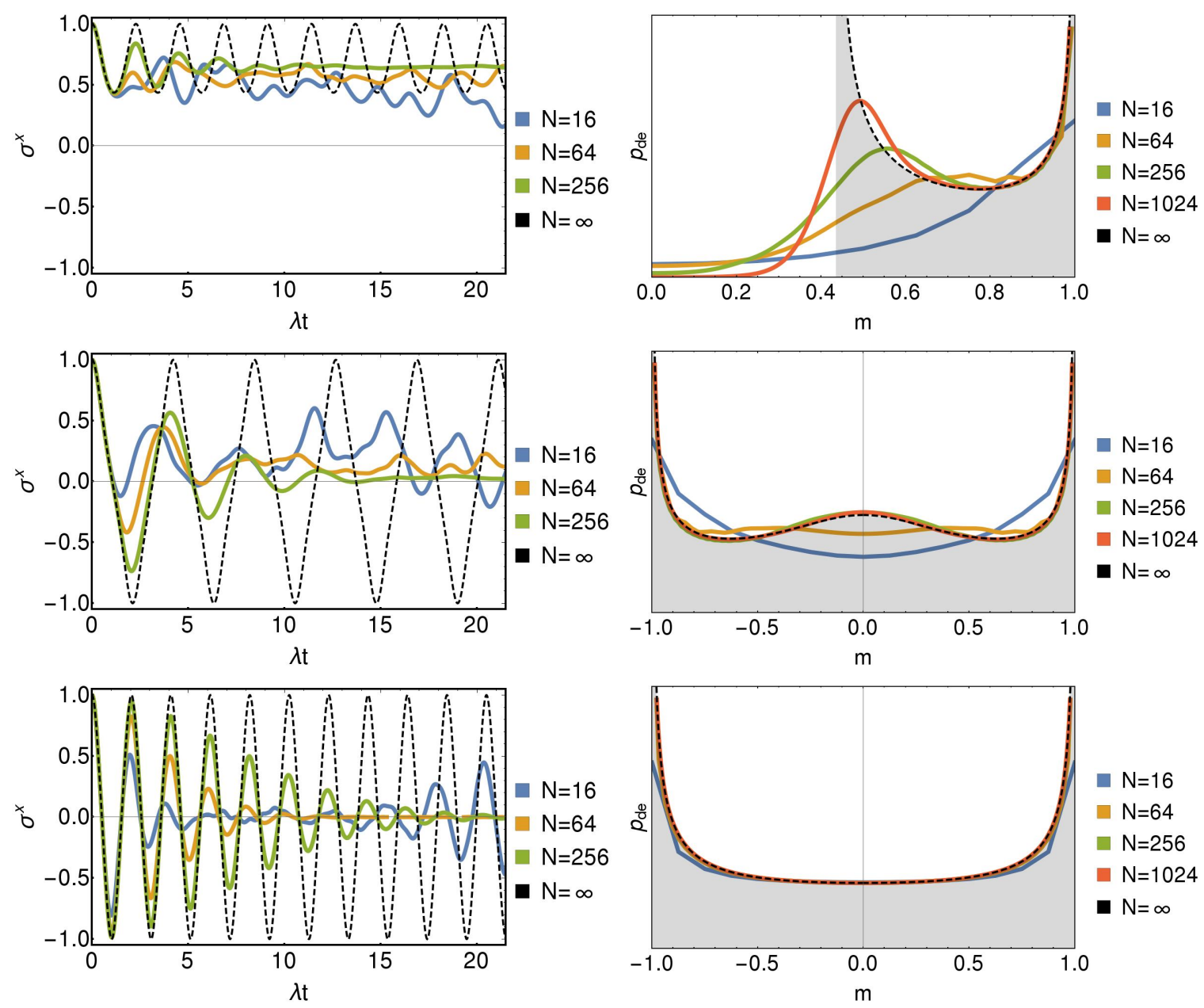

Figure 6. [Color online] Convergence to the classical behavior in the thermodynamic limit $N \rightarrow \infty$ of the quantum dynamics governed by the Hamiltonian (2) with finite size $N$ and with $s=1 / 2$. This is studied via exact diagonalization in the maximal spin sector. Left panels: Evolution of the dynamical order parameter with $g / \lambda=0.9$ (top), $g / \lambda=1.1($ center $), g / \lambda=1.7$ (bottom), and increasing system size $N=16,64,256$, starting from a fully polarized state along the $\hat{x}$-direction, i.e., from a ground state with $g_{0}=0$. The classical limit is shown by the black dashed curve. Right panels: corresponding infinite-time average distribution of the order parameter, as obtained from the diagonal ensemble $p_{\text {de }}(m)=\overline{|\langle\Psi(t) \mid m\rangle|^{2}}$, where $|m\rangle$ is the state with magnetization $m$ and the overline stands for infinite-time-average. The classical "microcanonical" distributions, obtained by averaging over the trajectory of $\mathcal{H}_{\mathrm{cl}}$ with energy $E=\left\langle\psi_{0}|H| \psi_{0}\right\rangle / N=-\lambda$, are shown by the black dashed curve. Note that the quantum evolution agrees with its classical limit over a time window that increases with $N$. After this time, quantum phenomena emerge. In all cases, damping of the classical oscillations takes place as a consequence of the quantum spreading of the wavepacket. Furthermore, for system sizes $N$ as small as 16, additional quantum effects become observable. In the top left panel, quantum tunneling to the opposite well can be observed in the dynamical ferromagnetic phase at relatively small time, which scales as $T_{\text {tun }}=\mathcal{O}\left(e^{c N}\right)$; note that the corresponding infinite-time distribution of the magnetization is suppressed in the classically forbidden region $m \approx 0$ as $N \rightarrow \infty$. In the center left panel, a remnant of ferromagnetic behavior can be observed in the dynamical paramagnetic phase, due to contributions to the wavepacket coming from ferromagnetic initial conditions (in order to visualize this, one should replace the small black dot in Fig. 4 with an extended circle of radius $1 / \sqrt{N}$ ). In the bottom left panel, recurrences in the evolution of the order parameter emerge at relatively small time $T_{\text {rec }}=\mathcal{O}(N)$, due to wavepacket refocusing after spreading. All these three effects occur at larger times for $N=64,256$, and thus do not appear in the relative plots.

relevance and consequences of these finite-size effects, we report in Fig. 6 the time evolution of the order parameter $\sigma^{x}$ as well as the infinite-time averaged distribution $p_{\text {de }}$ of the magnetization for increasing system sizes $N$, as obtained from numerical diagonalization of the Hamiltonian (2) in the maximal spin sector.

\section{STATIC AND DYNAMICAL SPIN-WAVE EXPANSIONS}

The lack of interaction between the collective mode discussed in Sec. II and the spin waves is an artifact of the infinite-range limit. In any realistic model, quantum 
fluctuations contribute to the dynamics and, as a result of this interaction, the system is expected to eventually thermalize. It is thus natural to investigate the possible persistence of instances of dynamical criticality discussed above in the pre-thermal stage of the dynamics, together with the possible onset of qualitatively new phenomena generated by these additional fluctuations. For this aim, we present in this Section a method to account systematically for the effect of fluctuations on the dynamics of general interacting spin models, which was briefly introduced in Ref. 66.

\section{A. Perturbative corrections to the equilibrium transition}

In order to understand the impact of quantum fluctuations on the physics of the fully-connected Ising ferromagnet of Sec. II, we consider perturbations in the form of additional spatially-decaying interactions,

$$
H=-\frac{\lambda}{N} \sum_{\mathbf{r}, \mathbf{r}^{\prime}} \sigma_{\mathbf{r}}^{x} \sigma_{\mathbf{r}^{\prime}}^{x}-g \sum_{\mathbf{r}} \sigma_{\mathbf{r}}^{z}-\sum_{\mathbf{r}, \mathbf{r}^{\prime}} J_{\left|\mathbf{r}-\mathbf{r}^{\prime}\right|} \sigma_{\mathbf{r}}^{x} \sigma_{\mathbf{r}^{\prime}}^{x},
$$

where $\mathbf{r}, \mathbf{r}^{\prime}$ run over a $d$-dimensional lattice with $N$ sites, and the coupling $J_{r}$ decays to zero upon increasing the geometrical distance $r=\left|\mathbf{r}-\mathbf{r}^{\prime}\right|$. For simplicity we will focus on the one-dimensional case $d=1$ with periodic boundary conditions, even though all of the results we find do not rely on this assumption, as will become clear in the following. Accordingly, we denote by $i, j=1, \ldots, N$ the lattice sites.

The perturbation makes the Hamiltonian a function not only of the spin Fourier component at $k=0$ (as occurs for $J_{r} \equiv 0$ ), but of all the Fourier components with $k \neq 0$. When the perturbation is small, the amplitude of the modes with $k \neq 0$ is expected to be small, so that we can treat them perturbatively at the lowest non-trivial order corresponding to a quadratic approximation. In order to do so, we introduce canonical coordinates representing small fluctuations around the mean-field spin-coherent states by using a Holstein-Primakoff transformation relative to the direction of the average collective spin vector $\left\langle\tilde{\vec{\sigma}}_{k=0}\right\rangle$. Let us start by describing this approach in equilibrium. We first rewrite the Hamiltonian (20) in terms of Fourier components,

$$
H=-\frac{\bar{\lambda}}{N}\left(\tilde{\sigma}_{k=0}^{x}\right)^{2}-g \tilde{\sigma}_{k=0}^{z}-\frac{1}{N} \sum_{k \neq 0} \tilde{J}_{k} \tilde{\sigma}_{k}^{x} \tilde{\sigma}_{-k}^{x},
$$

where $\bar{\lambda} \equiv \lambda+\tilde{J}_{0}, \tilde{J}_{k}=\tilde{J}_{-k}=\sum_{r=0}^{N-1} e^{-i k r} J_{r}$ and $\tilde{\sigma}_{k}^{\alpha}=$ $\sum_{j} e^{-i k j} \sigma_{j}^{\alpha}$, where $k$ varies in the Brillouin zone. Let us now introduce a rotated reference frame $(\hat{X}, \hat{Y}, \hat{Z})$, whose components in the original fixed frame $(\hat{x}, \hat{y}, \hat{z})$ are parameterized by the polar angles $\theta$ and $\phi$ as

$$
\hat{X} \equiv\left(\begin{array}{c}
\cos \theta \cos \phi \\
\cos \theta \sin \phi \\
-\sin \theta
\end{array}\right), \hat{Y} \equiv\left(\begin{array}{c}
-\sin \phi \\
\cos \phi \\
0
\end{array}\right), \hat{Z} \equiv\left(\begin{array}{c}
\sin \theta \cos \phi \\
\sin \theta \sin \phi \\
\cos \theta
\end{array}\right) .
$$

The spins can be then decomposed on the basis of $\mathcal{R}$ as

$$
\vec{\sigma}_{j}=\hat{X} \sigma_{j}^{X}+\hat{Y} \sigma_{j}^{Y}+\hat{Z} \sigma_{j}^{Z} .
$$

Accordingly, the Hamiltonian (21) can be rewritten as

$$
\begin{aligned}
\frac{H}{N}= & -\bar{\lambda}\left[(\hat{X} \cdot \hat{x}) \frac{\tilde{\sigma}_{0}^{X}}{N}+(\hat{Y} \cdot \hat{x}) \frac{\tilde{\sigma}_{0}^{Y}}{N}+(\hat{Z} \cdot \hat{x}) \frac{\tilde{\sigma}_{0}^{Z}}{N}\right]^{2} \\
& -g\left[(\hat{X} \cdot \hat{z}) \frac{\tilde{\sigma}_{0}^{X}}{N}+(\hat{Y} \cdot \hat{z}) \frac{\tilde{\sigma}_{0}^{Y}}{N}+(\hat{Z} \cdot \hat{z}) \frac{\tilde{\sigma}_{0}^{Z}}{N}\right] \\
& -\sum_{k \neq 0} \tilde{J}_{k}\left[(\hat{X} \cdot \hat{x}) \frac{\tilde{\sigma}_{k}^{X}}{N}+(\hat{Y} \cdot \hat{x}) \frac{\tilde{\sigma}_{k}^{Y}}{N}+(\hat{Z} \cdot \hat{x}) \frac{\tilde{\sigma}_{k}^{Z}}{N}\right] \cdot\left[(\hat{X} \cdot \hat{x}) \frac{\tilde{\sigma}_{-k}^{X}}{N}+(\hat{Y} \cdot \hat{x}) \frac{\tilde{\sigma}_{-k}^{Y}}{N}+(\hat{Z} \cdot \hat{x}) \frac{\tilde{\sigma}_{-k}^{Z}}{N}\right]
\end{aligned}
$$

in terms of the Fourier transforms $\tilde{\sigma}_{k}^{X, Y, Z}$ of $\sigma_{j}^{X, Y, Z}$.

In the rotated frame $\mathcal{R}$, we introduce the spin wave canonical variables via the Holstein-Primakoff transformation $^{80}$, expanded to lowest order in $1 / \sqrt{s}$, i.e.,

$$
\left\{\begin{array}{l}
\sigma_{j}^{X}=\frac{q_{j}}{\sqrt{s}}+\ldots \\
\sigma_{j}^{Y}=\frac{p_{j}}{\sqrt{s}}+\ldots \\
\sigma_{j}^{Z}=1-\frac{n_{j}}{s} \equiv 1-\frac{q_{j}^{2}+p_{j}^{2}-1}{2 s}
\end{array}\right.
$$

where $q_{j}$ and $p_{j}$ are the conjugate canonical variables representing small deviations of the spin away from the $\hat{Z}$-axis, and along the directions $\hat{X}$ and $\hat{Y}$, respectively. In our notation, the bosonic number operator, $n_{j}=b_{j}^{\dagger} b_{j}$, is defined via $b_{j}=\left(q_{j}+i p_{j}\right) / \sqrt{2}$. Accordingly, after introducing the coordinates $\tilde{q}_{k}=N^{-1 / 2} \sum_{j} e^{-i k j} q_{j}$ and $\tilde{p}_{k}=N^{-1 / 2} \sum_{j} e^{-i k j} p_{j}$ in Fourier space we get 


$$
\left\{\begin{array}{l}
\frac{\tilde{\sigma}_{k}^{X}}{N}=\frac{\tilde{q}_{k}}{\sqrt{N s}}+\ldots \\
\frac{\tilde{\sigma}_{k}^{Y}}{N}=\frac{\tilde{p}_{k}}{\sqrt{N s}}+\ldots \\
\frac{\tilde{\sigma}_{k}^{Z}}{N}=\delta_{k, 0}-\sum_{k^{\prime}} \frac{\tilde{q}_{k^{\prime}} \tilde{q}_{k-k^{\prime}}+\tilde{p}_{k^{\prime}} \tilde{p}_{k-k^{\prime}}-\delta_{k, 0}}{2 N s}
\end{array}\right.
$$

The Hamiltonian (24) can now be written in terms of the canonical spin wave coordinates,

$$
\begin{aligned}
H= & -\bar{\lambda} N(\hat{Z} \cdot \hat{x})^{2}\left(1-\frac{n_{0}+N_{\mathrm{sw}}}{N s}\right)^{2}-g N(\hat{Z} \cdot \hat{z})\left(1-\frac{n_{0}+N_{\mathrm{sw}}}{N s}\right) \\
& -2 \bar{\lambda} \frac{\sqrt{N}}{\sqrt{s}}(\hat{Z} \cdot \hat{x})\left(1-\frac{n_{0}+N_{\mathrm{sw}}}{N s}\right)\left[(\hat{X} \cdot \hat{x}) \tilde{q}_{0}+(\hat{Y} \cdot \hat{x}) \tilde{p}_{0}\right] \\
& -g \frac{\sqrt{N}}{\sqrt{s}}\left[(\hat{X} \cdot \hat{z}) \tilde{q}_{0}+(\hat{Y} \cdot \hat{z}) \tilde{p}_{0}\right] \\
& -\frac{\bar{\lambda}}{s}\left[(\hat{X} \cdot \hat{x})^{2} \tilde{q}_{0}^{2}+(\hat{Y} \cdot \hat{x})^{2} \tilde{p}_{0}^{2}+2(\hat{X} \cdot \hat{x})(\hat{Y} \cdot \hat{x}) \frac{\tilde{q}_{0} \tilde{p}_{0}+\tilde{p}_{0} \tilde{q}_{0}}{2}\right] \\
& +U_{2}+U_{3}+U_{4},
\end{aligned}
$$

with the $k \neq 0$ contribution of the short-range interaction split into the three terms:

$$
\begin{aligned}
& U_{2}=-\sum_{k \neq 0} \frac{\tilde{J}_{k}}{s}\left[(\hat{X} \cdot \hat{x})^{2} \tilde{q}_{k} \tilde{q}_{-k}+(\hat{Y} \cdot \hat{x})^{2} \tilde{p}_{k} \tilde{p}_{-k}\right. \\
&\left.+2(\hat{X} \cdot \hat{x})(\hat{Y} \cdot \hat{x}) \frac{\tilde{q}_{k} \tilde{p}_{-k}+\tilde{p}_{k} \tilde{q}_{-k}}{2}\right], \\
& U_{3}=+\frac{1}{\sqrt{N s}} \sum_{k \neq 0} \frac{\tilde{J}_{k}}{s}(\hat{Z} \cdot \hat{x}) \\
& \times\left\{(\hat{X} \cdot \hat{x})\left[\tilde{q}_{k} \sum_{k^{\prime}} \frac{\tilde{q}_{k^{\prime}} \tilde{q}_{-k-k^{\prime}}+\tilde{p}_{k^{\prime}} \tilde{p}_{-k-k^{\prime}}}{2}+(k \leftrightarrow-k)\right]+\right. \\
&\left.+(\hat{Y} \cdot \hat{x})\left[\tilde{p}_{k} \sum_{k^{\prime}} \frac{\tilde{q}_{k^{\prime}} \tilde{q}_{-k-k^{\prime}}+\tilde{p}_{k^{\prime}} \tilde{p}_{-k-k^{\prime}}}{2}+(k \leftrightarrow-k)\right]\right\}, \\
& U_{4}=-\frac{1}{N s} \sum_{k \neq 0} \frac{\tilde{J}_{k}}{s}(\hat{Z} \cdot \hat{x})^{2} \sum_{k^{\prime}} \frac{\tilde{q}_{k^{\prime}} \tilde{q}_{k-k^{\prime}}+\tilde{p}_{k^{\prime}} \tilde{p}_{k-k^{\prime}}}{2} \sum_{k^{\prime \prime}} \frac{\tilde{q}_{k^{\prime \prime}} \tilde{q}_{-k-k^{\prime \prime}}+\tilde{p}_{k^{\prime \prime}} \tilde{p}_{-k-k^{\prime \prime}}}{2},
\end{aligned}
$$

standing for the quadratic, cubic, and quartic terms in the spin waves, respectively.

In Eq. (27), the quantity $N_{\mathrm{sw}}$ is the total number of spin waves, i.e.,

$$
N_{\mathrm{sw}}=\sum_{k \neq 0} n_{k}=\sum_{k \neq 0} \frac{\tilde{q}_{k} \tilde{q}_{-k}+\tilde{p}_{k} \tilde{p}_{-k}-1}{2}
$$

[cf. Eq. (15)]. The expansion in Eq. (27) is valid as long as the spin waves have a low density $N_{\mathrm{sw}} \ll N s$, i.e., the collective spin magnitude is close to its maximal value $N s$. In this regime, spin waves behave as free bosonic excitations which interact with the macroscopic collective spin only, corresponding to the $k=0$ mode. Higher-order terms, which account for non-linear scattering among the spin waves, can be neglected: they are expected to contribute significantly to the dynamics only at longer times and to drive the system away from the pre-thermal regime relevant for the DPT discussed here.

Our approach is equivalent to treating fluctuations within the Gaussian approximation, which is the lowest non-trivial order beyond mean-field. This is expected to be sufficiently accurate when the interaction $\tilde{J}_{k \neq 0}$ introduces a small perturbation to the mean-field dynamics, such that a small spin-wave density $N_{\mathrm{sw}} /(\mathrm{Ns})$ is generated during the dynamics. In this case, similarly to the well-known Bogolyubov theory of weakly-interacting Bose gases ${ }^{81}$, we can treat them as free particles. Accordingly, the only relevant interaction is that between the 
collective mode $\tilde{q}_{0}, \tilde{p}_{0}$ and the spin waves, given by terms in $U_{3}$, which describe scattering of the zero-momentum mode into a pair of spin waves with opposite momenta $(k,-k)$, and viceversa. This approximation amounts to neglecting terms of order $\mathcal{O}\left(\left\langle N_{\text {sw }}\right\rangle / N s\right)^{2}$. Thereby, we arrive at the following form of the Hamiltonian (20), truncated to linear order in the collective $k=0$ mode and to quadratic order in the spin-wave fluctuations with $k \neq 0$,

$$
\begin{aligned}
& H \simeq-\bar{\lambda} N(\hat{Z} \cdot \hat{x})^{2}-g N(\hat{Z} \cdot \hat{z}) \\
& +\frac{1}{s} \sum_{k \neq 0}\left[2 \bar{\lambda}(\hat{Z} \cdot \hat{x})^{2}+g(\hat{Z} \cdot \hat{z})\right] \frac{\tilde{q}_{k} \tilde{q}_{-k}+\tilde{p}_{k} \tilde{p}_{-k}-1}{2} \\
& -\frac{1}{s} \sum_{k \neq 0} \tilde{J}_{k}\left[(\hat{X} \cdot \hat{x})^{2} \tilde{q}_{k} \tilde{q}_{-k}+(\hat{Y} \cdot \hat{x})^{2} \tilde{p}_{k} \tilde{p}_{-k}\right. \\
& \left.\quad+2(\hat{X} \cdot \hat{x})(\hat{Y} \cdot \hat{x}) \frac{\tilde{q}_{k} \tilde{p}_{-k}+\tilde{p}_{k} \tilde{q}_{-k}}{2}\right] \\
& +\frac{\sqrt{N}}{\sqrt{s}} \tilde{q}_{0}\left\{-2 \bar{\lambda}\left(1-\frac{N_{\mathrm{sw}}}{N s}\right)(\hat{Z} \cdot \hat{x})(\hat{X} \cdot \hat{x})-g(\hat{X} \cdot \hat{z})\right. \\
& \left.\quad+2(\hat{Z} \cdot \hat{x}) \frac{1}{N s} \sum_{k \neq 0} \tilde{J}_{k}\left[(\hat{X} \cdot \hat{x}) \tilde{q}_{k} \tilde{q}_{-k}+(\hat{Y} \cdot \hat{x}) \frac{\tilde{q}_{k} \tilde{p}_{-k}+\tilde{p}_{k} \tilde{q}_{-k}}{2}\right]\right\} \\
& +\frac{\sqrt{N}}{\sqrt{s}} \tilde{p}_{0}\left\{-2 \bar{\lambda}\left(1-\frac{N_{\mathrm{sw}}}{N s}\right)(\hat{Z} \cdot \hat{x})(\hat{Y} \cdot \hat{x})-g(\hat{Y} \cdot \hat{z})\right. \\
& \left.\quad+2(\hat{Z} \cdot \hat{x}) \frac{1}{N s} \sum_{k \neq 0} \tilde{J}_{k}\left[(\hat{Y} \cdot \hat{x}) \tilde{p}_{k} \tilde{p}_{-k}+(\hat{X} \cdot \hat{x}) \frac{\tilde{q}_{k} \tilde{p}_{-k}+\tilde{p}_{k} \tilde{q}_{-k}}{2}\right]\right\},
\end{aligned}
$$

where the explicit expressions of the various scalar products between versors in terms of the rotation angles $\theta$ and $\phi$ can be inferred from Eq. (22). The Hamiltonian (30) is our starting point for assessing the impact of fluctuations on the equilibrium and dynamical phase transition occurring in the LMG model.

We first study the equilibrium behavior in the presence of fluctuations. The average total spin in equilibrium can be determined at the Gaussian level by imposing vanishing expectation values of $\tilde{q}_{0}$ and $\tilde{p}_{0}$, i.e.,

$$
\left\langle\tilde{q}_{0}\right\rangle=\left\langle\tilde{p}_{0}\right\rangle=0 .
$$

Equation (26) with $k=0$ shows that this is equivalent to requiring that the average total spin $\left\langle\tilde{\vec{\sigma}}_{k=0}\right\rangle$ is aligned along the $\hat{Z}$-direction determined by the spherical angles $\theta, \phi$. In the mean-field limit $\tilde{J}_{k \neq 0}=0$, the spin waves are frozen in their vacuum state and the problem becomes equivalent to finding the ground state of the single classical spin $\vec{\sigma}=\left\langle\tilde{\vec{\sigma}}_{k=0}\right\rangle / N$ on the sphere. As $\tilde{J}_{k \neq 0} \neq 0$, the spin waves are generically excited even in the ground state, analogously to the depletion of the condensate fraction in a dilute Bose gas in the presence of weak inter- actions. Eqs. (31) are actually satisfied when the values of $\theta$ and $\phi$ are chosen in such a way that the equilibrium expectation values of the two curly brackets in Eq. (30) vanish. The second one does it if $\hat{Y} \cdot \hat{x}=\hat{Y} \cdot \hat{z}=0$, and

$$
\sum_{k \neq 0} \tilde{J}_{k}\left\langle\frac{\tilde{q}_{k} \tilde{p}_{-k}+\tilde{p}_{k} \tilde{q}_{-k}}{2}\right\rangle=0,
$$

which implies that $\phi^{*}=0$ or $\pi$, meaning that the collective spin lies in the $x z$-plane, as could be anticipated based on symmetry arguments. The remaining equation determines the value of $\theta^{*}$. In particular, $\theta^{*}=0$ is always a solution: however, it is stable only for $g$ large enough. For small $g$, stable solutions are calculated as follows. First we diagonalize the quadratic part of the Hamiltonian [second and third sums on the r.h.s. of Eq. (30)] obtaining a parametric spin wave dispersion relation $\omega_{k} / s$,

$$
\omega_{k}=\sqrt{\left(2 \bar{\lambda} \sin ^{2} \theta+g \cos \theta\right)\left(2 \bar{\lambda} \sin ^{2} \theta+g \cos \theta-2 \tilde{J}_{k} \cos ^{2} \theta\right)} .
$$

Denoting by $\omega_{k}^{(0)} / s$ the "unperturbed" common frequency of the spin wave modes,

$$
\omega_{k}^{(0)} \equiv \omega^{(0)}=2 \bar{\lambda} \sin ^{2} \theta+g \cos \theta
$$


the zero-temperature Gaussian expectation values of the relevant observables can then be expressed as

$$
\left\{\begin{aligned}
\left\langle\tilde{q}_{k} \tilde{q}_{-k}\right\rangle & =\frac{1}{2} \frac{\omega_{k}^{(0)}}{\omega_{k}}, \\
\left\langle\tilde{p}_{k} \tilde{p}_{-k}\right\rangle & =\frac{1}{2} \frac{\omega_{k}}{\omega_{k}^{(0)}}, \\
\left\langle\frac{\tilde{q}_{k} \tilde{p}_{-k}+\tilde{p}_{k} \tilde{q}_{-k}}{2}\right\rangle & =0 .
\end{aligned}\right.
$$

Accordingly, the equation of state which determines $\theta^{*}$ reads

$$
\sin \theta^{*}\left[-2 \bar{\lambda}(1-\epsilon) \cos \theta^{*}+g+\cos \theta^{*} \frac{1}{N s} \sum_{k \neq 0} \tilde{J}_{k} \sqrt{\frac{2 \bar{\lambda} \sin ^{2} \theta^{*}+g \cos \theta^{*}}{2 \bar{\lambda} \sin ^{2} \theta^{*}+g \cos \theta^{*}-2 \tilde{J}_{k} \cos ^{2} \theta^{*}}}\right]=0 .
$$

Combining Eqs. (29) and (35), we get an explicit expression for the total spin depletion $\epsilon$ defined by the equation

$$
\frac{\left|\left\langle\tilde{\vec{\sigma}}_{k=0}\right\rangle\right|}{N}=1-\frac{\left\langle N_{\mathrm{sw}}\right\rangle}{N s} \equiv 1-\epsilon
$$

i.e.,

$$
\epsilon \equiv \frac{\left\langle N_{\mathrm{sw}}\right\rangle}{N s}=\frac{1}{2 N s} \sum_{k \neq 0}\left(\frac{1}{2} \frac{\omega_{k}^{(0)}}{\omega_{k}}+\frac{1}{2} \frac{\omega_{k}}{\omega_{k}^{(0)}}-1\right)_{\theta=\theta^{*}} .
$$

Note that $\epsilon \geq 0$ and $\epsilon=\mathcal{O}\left(\tilde{J}_{k \neq 0}^{2}\right)$. Moreover, in the limits $g \rightarrow 0$ and $g \rightarrow \infty$, the depletion $\epsilon$ at equilibrium vanishes, whereas it is arguably maximal at the critical point $g_{\text {cr }}=2 \bar{\lambda}-\mathcal{O}\left(\tilde{J}_{k \neq 0}^{2}\right)$ (see below).

As a check, in the mean-field case $\tilde{J}_{k \neq 0} \equiv 0$, Eqs. (33) and (36) imply $\omega_{k}^{(0)}=\omega_{k}, \epsilon=0$, and $\cos \theta^{*}=g / 2 \lambda$ in the ferromagnetic phase $g<2 \lambda$, retrieving the mean-field equilibrium properties. As soon as a spatially-decaying interaction $\tilde{J}_{k \neq 0} \neq 0$ is turned on, quantum fluctuations modify the equilibrium state.

In the equilibrium paramagnetic phase $g>g_{\mathrm{cr}}$, the ground state has $\theta^{*}=0$, and from Eq. (33) we find

$$
\omega_{k,>}=\sqrt{g\left(g-2 \tilde{J}_{k}\right)} .
$$

Deep in the equilibrium ferromagnetic phase, with $g \rightarrow 0$, the system approaches instead a full ferromagnetic ordering with $\theta^{*} \rightarrow \pi / 2$, and therefore the corresponding dispersion relation derived from Eq. (33) becomes independent of $k$, i.e. the band becomes flat,

$$
\omega_{k,<} \underset{g \rightarrow 0}{\longrightarrow} 2 \bar{\lambda} .
$$

This could have been anticipated by observing that in the limit $g \rightarrow 0$ the spin Hamiltonian (20) becomes diagonal in the $\sigma^{x}$-basis.

Let us determine now the perturbative corrections to the critical point, employing an equivalent variational approach. The critical point corresponds to the value of $g$ at which the paramagnetic configuration $\theta=0$ becomes an unstable saddle-point of $H$. We compute the variational energy $\mathcal{E}(\theta)=\langle H\rangle_{\theta}$ as a function of $\theta$ (with fixed $\phi=0$ ), by taking the average of $H$ in Eq. (30) with $\left\langle\tilde{q}_{0}\right\rangle=\left\langle\tilde{p}_{0}\right\rangle=0$ and $\left\langle\tilde{q}_{k} \tilde{q}_{-k}\right\rangle$ given by Eq. (35), thereby obtaining

$$
\frac{\mathcal{E}(\theta)}{N}=-\bar{\lambda} \sin ^{2} \theta-g \cos \theta+\frac{1}{N s} \sum_{k \neq 0} \frac{\omega_{k}-\omega_{k}^{(0)}}{2} .
$$

In order to determine the stability of the solution $\theta^{*}=0$, we expand $\sim \mathcal{E}(\theta)$ at small $\theta$, finding

$$
\begin{aligned}
\frac{\mathcal{E}(\theta)}{N} \underset{\theta \rightarrow 0}{\sim} & -g+\frac{1}{N s} \sum_{k \neq 0} \frac{1}{2}\left(\sqrt{g\left(g-2 \tilde{J}_{k}\right)}-g\right) \\
& +\left\{g-2 \bar{\lambda}+\frac{1}{N s} \sum_{k \neq 0}\left[\sqrt{g\left(g-2 \tilde{J}_{k}\right)} \frac{1}{2}\left(\frac{2 \bar{\lambda}-g / 2+2 \tilde{J}_{k}}{g-2 \tilde{J}_{k}}+\frac{2 \bar{\lambda}-g / 2}{g}\right)-(2 \bar{\lambda}-g / 2)\right]\right\} \frac{\theta^{2}}{2}+\mathcal{O}\left(\theta^{4}\right) .
\end{aligned}
$$

The critical point is then determined by the vanishing of the coefficient of the quadratic term in curly bracket, which yields an equation for $g_{\mathrm{cr}}$. The corrections for small
$\tilde{J}_{k \neq 0}$ may be found perturbatively by expanding the solution $g_{\mathrm{cr}}\left(\tilde{J}_{k \neq 0}\right)$ with respect to $\tilde{J}_{k \neq 0}$ and by equating both sides order by order. The explicit calculation yields 
a quadratic correction:

$$
g_{\mathrm{cr}}=2 \bar{\lambda}\left\{1-\frac{5}{16} \frac{1}{N s} \sum_{k \neq 0}\left(\frac{\tilde{J}_{k}}{\bar{\lambda}}\right)^{2}\right\}+\mathcal{O}\left(\tilde{J}_{k \neq 0}^{3}\right) .
$$

As expected on physical grounds, the spin waves destabilize the ferromagnetic ordering and thereby lower the critical value $g_{\mathrm{cr}}$.

The ground state equations discussed above can immediately be generalized to the case with a finite temperature $T>0$. In fact, it is sufficient to substitute in Eq. (35) the pre-factor $1 / 2$ with

$$
\frac{1}{2}+\left\langle n_{k}\right\rangle_{T}=\frac{1}{2}+\frac{1}{e^{\omega_{k} / T}-1},
$$

where $\left\langle n_{k}\right\rangle_{T}$ is the Bose-Einstein distribution of the excited spin waves. The expression of $\epsilon$ in Eq. (38) and the equation of state (36) are modified accordingly. As in the mean-field case, thermal corrections are exponentially suppressed at low temperature as long as the gap in the dispersion relation (33) is non-vanishing.

\section{B. Dynamics: Time-dependent spin wave theory}

The non-equilibrium dynamics in the presence of weak fluctuations can be analyzed by generalizing the approach developed in the previous section to a time-evolving state. The spin wave expansion will be performed with respect to a time-dependent rotated frame $\mathcal{R}$, with the angles $\theta(t), \phi(t)$ co-moving with the average collective $\operatorname{spin}^{66}$. This is implemented by the unitary operator

$$
V(\theta(t), \phi(t))=e^{-i \phi s \sum_{j} \sigma_{j}^{z}} e^{-i \theta s \sum_{j} \sigma_{j}^{y}},
$$

acting on the spins as a time-dependent global rotation:

$$
\left\{\begin{array}{l}
V \sigma_{j}^{x} V^{\dagger}=\hat{X} \cdot \vec{\sigma}_{j} \equiv \sigma_{j}^{X}, \\
V \sigma_{j}^{y} V^{\dagger}=\hat{Y} \cdot \vec{\sigma}_{j} \equiv \sigma_{j}^{Y}, \\
V \sigma_{j}^{z} V^{\dagger}=\hat{Z} \cdot \vec{\sigma}_{j} \equiv \sigma_{j}^{Z} .
\end{array}\right.
$$

The Heisenberg equations of motion for $\sigma_{j}^{\alpha}(\alpha \in$ $\{X, Y, Z\})$, in the mobile frame $\mathcal{R}$, read then

$$
\frac{d}{d t} \sigma_{j}^{\alpha}=\frac{1}{i}\left[\sigma_{j}^{\alpha}, \widetilde{H}\right], \quad \text { with } \widetilde{H} \equiv H+i V \dot{V}^{\dagger},
$$

where the last term is the inertial force contribution equal to

$$
i V \dot{V}^{\dagger}=-s \vec{\omega}(t) \cdot \sum_{j} \vec{\sigma}_{j}=-s \vec{\omega}(t) \cdot \tilde{\vec{\sigma}}_{0}
$$

where we introduced the vector $\vec{\omega}=\left(\omega^{X}, \omega^{Y}, \omega^{Z}\right)$, with $\omega^{X}=\dot{\hat{Y}} \cdot \hat{Z}, \omega^{Y}=\dot{\hat{Z}} \cdot \hat{X}$, and $\omega^{Z}=\dot{\hat{X}} \cdot \hat{Y}$.

The resulting Hamiltonian $\widetilde{H}(t)$ is thus given by the expression in Eq. (30) for $H$ with time-dependent
$\hat{X}(t), \hat{Y}(t), \hat{Z}(t)$ [i.e., with time-dependent $\theta(t), \phi(t)]$, and with the additional terms

$$
\begin{aligned}
-s(\dot{\hat{X}} \cdot \hat{Y}) \tilde{\sigma}_{0}^{Z}= & -s(\dot{\hat{X}} \cdot \hat{Y}) \\
& +\frac{1}{s} \sum_{k \neq 0}[s(\dot{\hat{X}} \cdot \hat{Y})] \frac{\tilde{q}_{k} \tilde{q}_{-k}+\tilde{p}_{k} \tilde{p}_{-k}-1}{2}, \\
-s(\dot{\hat{Y}} \cdot \hat{Z}) \tilde{\sigma}_{0}^{X}= & \frac{\sqrt{N}}{\sqrt{s}} \tilde{q}_{0}\{-s(\dot{\hat{Y}} \cdot \hat{Z})\}, \\
-s(\dot{\hat{Z}} \cdot \hat{X}) \tilde{\sigma}_{0}^{Y}= & \frac{\sqrt{N}}{\sqrt{s}} \tilde{p}_{0}\{-s(\dot{\hat{Z}} \cdot \hat{X})\},
\end{aligned}
$$

to be added to the second, fourth and fifth line of Eq. (30), respectively. This time-dependent Hamiltonian governs the self-consistent coupled evolution equations of the angles $\theta, \phi$ and of the excitations $\tilde{q}_{k}, \tilde{p}_{k}$. In particular, the motion of the angles is obtained by imposing the condition (31) to hold at all times, which corresponds to setting the coefficients of $\tilde{q}_{0}$ and $\tilde{p}_{0}$ in $\widetilde{H}(t)$ equal to zero. This procedure yields a pair of classical evolution equations for $\theta(t), \phi(t)$, which depend also on the spin-wave correlation functions. The excitation of spin waves thereby affects the mean-field trajectory of $\theta(t), \phi(t)$ at order $\tilde{J}_{k \neq 0}$ for weak integrability breaking. Concurrently, the motion of the vacuum $\theta(t), \phi(t)$ drives the non-equilibrium evolution of the spin excitations $\tilde{q}_{k}, \tilde{p}_{k}$. Explicitly, we obtain

$$
\left\{\begin{aligned}
s \frac{d}{d t} \theta= & +2 \bar{\lambda}[1-\epsilon(t)] \sin \theta \cos \phi \sin \phi \\
& -2\left(\frac{1}{N s} \sum_{k \neq 0} \tilde{J}_{k} \Delta_{k}^{p p}(t)\right) \sin \theta \cos \phi \sin \phi \\
& +2\left(\frac{1}{N s} \sum_{k \neq 0} \tilde{J}_{k} \Delta_{k}^{q p}(t)\right) \cos \theta \sin \theta \cos ^{2} \phi \\
s \frac{d}{d t} \phi= & -g+2 \bar{\lambda}[1-\epsilon(t)] \cos \theta \cos ^{2} \phi \\
& -2\left(\frac{1}{N s} \sum_{k \neq 0} \tilde{J}_{k} \Delta_{k}^{q q}(t)\right) \cos \theta \cos ^{2} \phi \\
& +2\left(\frac{1}{N s} \sum_{k \neq 0} \tilde{J}_{k} \Delta_{k}^{q p}(t)\right) \sin \phi \cos \phi
\end{aligned}\right.
$$

where $\Delta_{k}^{q q}(t), \Delta_{k}^{q p}(t), \Delta_{k}^{q q}(t)$ are the equal-time correlation functions

$$
\left\{\begin{aligned}
\Delta_{k}^{q q}(t) & \equiv\left\langle\tilde{q}_{k}(t) \tilde{q}_{-k}(t)\right\rangle \\
\Delta_{k}^{p p}(t) & \equiv\left\langle\tilde{p}_{k}(t) \tilde{p}_{-k}(t)\right\rangle \\
\Delta_{k}^{q p}(t) & \equiv \frac{1}{2}\left\langle\tilde{q}_{k}(t) \tilde{p}_{-k}(t)+\tilde{p}_{k}(t) \tilde{q}_{-k}(t)\right\rangle,
\end{aligned}\right.
$$

and, as in Eq. (52), the non-equilibrium density $\epsilon(t)$ of spin waves reads

$$
\epsilon(t) \equiv \frac{1}{N s} \sum_{k \neq 0}\left\langle n_{k}(t)\right\rangle=\frac{1}{N s} \sum_{k \neq 0} \frac{\Delta_{k}^{q q}(t)+\Delta_{k}^{p p}(t)-1}{2} .
$$


Using now the equations of motion for the spin wave coordinates,

$$
\left\{\begin{array}{l}
s \frac{d}{d t} \tilde{q}_{k}=+2 \bar{\lambda} \cos ^{2} \phi \tilde{p}_{k}-2 \tilde{J}_{k} \sin ^{2} \phi \tilde{p}_{k}+2 \tilde{J}_{k} \cos \theta \cos \phi \sin \phi \tilde{q}_{k} \\
s \frac{d}{d t} \tilde{p}_{k}=-2 \bar{\lambda} \cos ^{2} \phi \tilde{q}_{k}+2 \tilde{J}_{k} \cos ^{2} \theta \cos ^{2} \phi \tilde{q}_{k}-2 \tilde{J}_{k} \cos \theta \cos \phi \sin \phi \tilde{p}_{k}
\end{array}\right.
$$

one obtains the evolution of the parameters $\Delta_{k}^{q q}, \Delta_{k}^{q p}, \Delta_{k}^{p p}$, which describe the dynamics of the Gaussian wavefunction of the spin waves:

$$
\left\{\begin{array}{l}
s \frac{d}{d t} \Delta_{k}^{q q}=4 \tilde{J}_{k} \cos \theta \cos \phi \sin \phi \Delta_{k}^{q q}+4\left(\bar{\lambda} \cos ^{2} \phi-\tilde{J}_{k} \sin ^{2} \phi\right) \Delta_{k}^{q p} \\
s \frac{d}{d t} \Delta_{k}^{q p}=-2\left(\bar{\lambda} \cos ^{2} \phi-\tilde{J}_{k} \cos ^{2} \theta \cos ^{2} \phi\right) \Delta_{k}^{q q}+2\left(\bar{\lambda} \cos ^{2} \phi-\tilde{J}_{k} \sin ^{2} \phi\right) \Delta_{k}^{p p} \\
s \frac{d}{d t} \Delta_{k}^{p p}=-4\left(\bar{\lambda} \cos ^{2} \phi-\tilde{J}_{k} \cos ^{2} \theta \cos ^{2} \phi\right) \Delta_{k}^{q p}-4 \tilde{J}_{k} \cos \theta \cos \phi \sin \phi \Delta_{k}^{p p}
\end{array}\right.
$$

Note that the evolution does not conserve the occupation numbers $\left\{n_{k}\right\}$, except in the mean-field limit $\tilde{J}_{k \neq 0}=0$. The equations of motion in Eq. (54) are actually not independent, as the quantities $\Delta^{q q}, \Delta^{q p}, \Delta^{p p}$ are related by the condition

$$
4\left(\Delta^{q p}\right)^{2}=4 \Delta^{q q} \Delta^{p p}-1
$$

which is an exact property of Gaussian quantum states with minimal uncertainty, and which is then satisfied at all times and for all values of $k$.

The dynamical problem is now fully specified by the system of $2 N$ coupled evolution equations (50) and (54), taking into account the constraints (55), together with suitable initial conditions, which can correspond to the ground state or to a thermal state of the pre-quench Hamiltonian. These equilibrium states, already determined in Sec. III A via the equation of state (36) (and its generalization to thermal states), may be retrieved by looking for stationary solutions of the dynamical equations with the initial parameters $g_{0}, \lambda_{0}, \tilde{J}_{k, 0}$. A variation in time of $g=g(t)$, corresponding to the driving under consideration, will then yield the non-equilibrium evolution at Gaussian level according to the dynamical equa- tions of motion derived above.

\section{Dynamics: Time-independent approach}

The system of coupled evolution equations for the collective spin and the spin waves discussed above can also be derived by using a time-independent approach. Indeed, the original Hamiltonian $H$ in Eq. (20) can be written in terms of two global canonical variables, the total spin projection $P$ along the $\hat{z}$-direction

$$
P=s \tilde{\sigma}_{k=0}^{z}=\left(N s-N_{\mathrm{sw}}\right) \cos \theta
$$

and its conjugated angle

$$
Q=\phi
$$

[see Eq. (12)], in addition to the canonical spin wave variables $\tilde{q}_{k}, \tilde{p}_{k}$ analogous to the ones introduced in the previous section [cf. Eq. (26)]. An explicit calculation shows that these observables provide a complete set of $2 N$ canonical variables for the spin system, i.e.,

$$
\left[Q, \tilde{q}_{k}\right]=\left[Q, \tilde{p}_{k}\right]=\left[P, \tilde{q}_{k}\right]=\left[P, \tilde{p}_{k}\right]=0 .
$$

Expanding $H$ up to the quadratic order in the modes $\tilde{q}_{k}$ and $\tilde{p}_{k}$, while retaining the full non-linearity in the collective spin coordinates $Q$ and $P$, one has ${ }^{82}$

$$
\begin{aligned}
H \simeq & -N g \frac{P}{N s}-N \bar{\lambda}\left[\left(1-2 \frac{N_{\mathrm{sw}}}{N s}\right)-\frac{P^{2}}{N^{2} s^{2}}\right] \cos ^{2} Q \\
& -\sum_{k \neq 0} \frac{\tilde{J}_{k}}{s}\left[\frac{P^{2}}{N^{2} s^{2}} \cos ^{2} Q \tilde{q}_{k} \tilde{q}_{-k}+\sin ^{2} Q \tilde{p}_{k} \tilde{p}_{-k}-2 \frac{P}{N s} \cos Q \sin Q \frac{\tilde{q}_{k} \tilde{p}_{-k}+\tilde{p}_{k} \tilde{q}_{-k}}{2}\right],
\end{aligned}
$$

where $N_{\text {sw }}$ is defined as in Eq. (29). Conceptually, this corresponds to promoting $\theta$ and $\phi$ in the Hamiltonian to 
proper dynamical variables rather than treating them as external parameters to be self-consistently adjusted, as was the case in the time-dependent approach discussed in the previous Section. Accordingly, in this derivation, there is no need to introduce the variables $\tilde{q}_{0}, \tilde{p}_{0}$.

The equations of motion derived from the timeindependent Hamiltonian (59) are

$$
\left\{\begin{aligned}
s \dot{Q}= & -g+2 \bar{\lambda} \frac{P}{N s} \cos ^{2} Q-\frac{1}{N s} \sum_{k \neq 0} 2 \tilde{J}_{k}\left[\frac{P}{N s} \cos ^{2} Q \Delta_{k}^{q q}-\cos Q \sin Q \Delta_{k}^{q p}\right], \\
s \frac{\dot{P}}{N s}= & -2 \bar{\lambda}\left[\left(1-2 \frac{N_{\mathrm{sw}}}{N s}\right)-\frac{P^{2}}{N^{2} s^{2}}\right] \cos Q \sin Q \\
& -\frac{1}{N s} \sum_{k \neq 0} 2 \tilde{J}_{k}\left[\frac{P^{2}}{N^{2} s^{2}} \cos Q \sin Q \Delta_{k}^{q q}-\cos Q \sin Q \Delta_{k}^{p p}+\frac{P}{N s}\left(\cos ^{2} Q-\sin ^{2} Q\right) \Delta_{k}^{q p}\right], \\
s \dot{\tilde{q}}_{k}= & +2 \bar{\lambda} \cos ^{2} Q \tilde{p}_{k}-2 \tilde{J}_{k} \sin ^{2} Q \tilde{p}_{k}+2 \tilde{J}_{k} \frac{P}{N s} \cos Q \sin Q \tilde{q}_{k}, \\
s \dot{\tilde{p}}_{k}= & -2 \bar{\lambda} \cos ^{2} Q \tilde{q}_{k}+2 \tilde{J}_{k} \frac{P^{2}}{N^{2} s^{2}} \cos ^{2} Q \tilde{q}_{k}-2 \tilde{J}_{k} \frac{P}{N s} \cos Q \sin Q \tilde{p}_{k},
\end{aligned}\right.
$$

where the $\Delta_{k}$ 's are defined as in Eq. (51).

Crucially, the quantum fluctuations of the collective operators $P / N$ and $Q$ in the initial state are of order $1 / \sqrt{N}$, and hence they behave like uncertainty-free classical variables in the thermodynamic limit (see Sec. II C for details on the convergence to the classical behavior). By identifying $Q=\phi$ and by changing variable from $P$ to $\theta$ via Eq. (56), after taking quantum averages with $\left\langle N_{\mathrm{sw}}\right\rangle /(N s) \equiv \epsilon$ [cf. Eq. (52)], one finds

$$
\dot{\theta}=\frac{\frac{\dot{P}}{N s}+\cos \theta \dot{\epsilon}}{-(1-\epsilon) \sin \theta}
$$

and one easily verifies that the equations of motion (60) obtained here are equivalent to Eqs. (50) and (53) obtained within the time-dependent spin wave approach, to the quadratic order in the quantum fluctuations.

The quadratic spin-wave expansion discussed in this Section indicates that the system with Hamiltonian (20) can be alternatively regarded as being composed by a macroscopic classical degree of freedom $(Q, P)$, corresponding to the collective spin, interacting with an extensive ensemble of quantum oscillators $\left\{\left(\tilde{q}_{k}, \tilde{p}_{k}\right)\right\}_{k \neq 0}$, see Eq. (59).

\section{IMPACT OF THE SPIN WAVES ON THE MEAN-FIELD DYNAMICAL PHASE TRANSITION}

Let us now apply the methods described in the previous section in order to study the impact of fluctuations on the mean-field dynamical criticality discussed in Sec. II. For the sake of definiteness, we will first depart from the exactly solvable mean-field limit by considering a model in one dimension where a nearest-neighbor interaction is added to the infinite-range interaction of the
LMG model ${ }^{66}$. A similar analysis is then carried out and similar results are obtained in Sec. IV B for a much wider class of models.

We thus consider the Hamiltonian

$$
H=-\frac{\lambda}{N} \sum_{i, j=1}^{N} \sigma_{i}^{x} \sigma_{j}^{x}-g \sum_{i=1}^{N} \sigma_{i}^{z}-J \sum_{i=1}^{N} \sigma_{i}^{x} \sigma_{i+1}^{x},
$$

where the strength of the nearest-neighbor perturbation is controlled by the parameter $J$ and periodic boundary conditions are understood. In the opposite limit $\lambda \rightarrow 0$ with finite $J$, the model reduces to the well-known quantum Ising chain in a transverse field, which is exactly solvable in terms of free Bogolubov fermions ${ }^{68}$. In this case, however, dynamical criticality disappears, as discussed in the Introduction.

In order to study the resulting dynamics, we will use Eqs. (50), (51), (52), (54), where $\tilde{J}_{k}=J \cos k$ with $k=$ $(2 \pi / N) j, j=-(N / 2)+1, \ldots,-1,0,1, \ldots,(N / 2)-1, N / 2$ for this periodic one-dimensional chain.

\section{A. Quench: Chaotic dynamical phase}

We numerically integrated the evolution equations (50) and (54) for a range of post-quench values of $g$ and $J$ and starting from a fully polarized ferromagnetic initial state with $\left\langle\sigma_{j}^{x}(t=0)\right\rangle=1$ (i.e., the pre-quench Hamiltonian is chosen with $g_{0}=0$, and the value of $J_{0}$ is thus actually immaterial as long as $\left|J_{0}\right|<\lambda$ ). At each integration time, we compute the time-dependent components of the average collective spin $\vec{\sigma}$,

$$
\vec{\sigma}(t) \equiv \frac{1}{N}\left\langle\tilde{\vec{\sigma}}_{k=0}(t)\right\rangle=[1-\epsilon(t)]\left(\begin{array}{c}
\sin \theta(t) \cos \phi(t) \\
\sin \theta(t) \sin \phi(t) \\
\cos \theta(t)
\end{array}\right),
$$




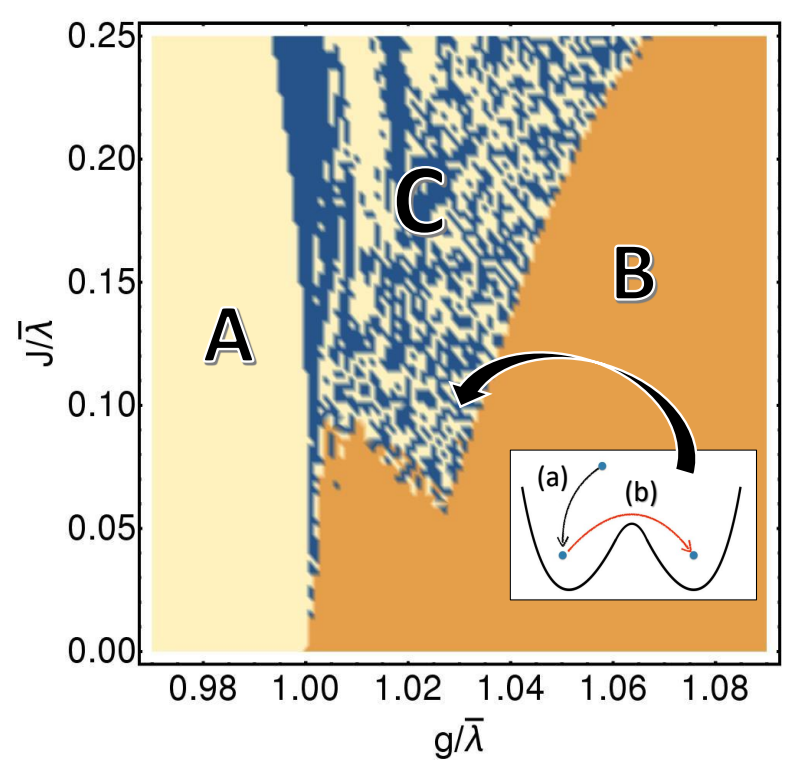

Figure 7. [Color online] Dynamical phase diagram of the model in Eq. (20) after a quench of the magnetic field $g_{0}=0 \rightarrow g$ starting from the fully polarized ground state with positive magnetization, as a function of $g$ and $J$. Here $N=100$. As energy scale we choose $\bar{\lambda} \equiv \lambda+J$. The color of each point of the diagram is determined by the sign of the long-time average $\overline{\sigma^{x}}$ of $\sigma^{x}(t)$ : light yellow for $\overline{\sigma^{x}}>0$, orange for $\overline{\sigma^{x}}=0$, and blue for $\overline{\sigma^{x}}<0$. Regions $\mathrm{A}$ and $\mathrm{B}$ are perturbative extensions of the dynamical ferromagnetic and paramagnetic phases of the LMG model with $J=0$, corresponding to the horizontal axis (see Fig. 8 for an illustration of the dynamics within A and B). Upon increasing $J$ at fixed $g$, in a neighborhood of the mean-field critical point $g=\bar{\lambda}$, a new chaotic dynamical ferromagnetic phase $\mathrm{C}$ emerges, within which the magnetization $\sigma^{x}(t)$, after an initial dynamical paramagnetic behavior, gets trapped in one of the two symmetry-broken sectors with opposite signs of the collective magnetization [process (a) in the inset], in some cases followed by hopping between them [process (b) in the inset] (see Fig. 9 for an illustration of the dynamics within C). The extent and features of the three phases A, B, C are stable as $N$ is increased.

verifying that the non-equilibrium density $\epsilon(t)$ of spin waves [see Eq. (52)] approaches asymptotically a small value at long times within the range of parameters considered. From this $\vec{\sigma}(t)$, we compute the long-time average of the magnetization along the ferromagnetic direction $\hat{x}$, i.e., the dynamical order parameter $\overline{\sigma^{x}}$, and plot it for different values of $J$ and $g$, coloring the corresponding point in light yellow if $\overline{\sigma^{x}}>0$ (dynamical ferromagnetic ordering in the initial sector), in orange if $\overline{\sigma^{x}}=0$ (dynamical paramagnetic behavior), and in blue if $\overline{\sigma^{x}}<0$ (reversed dynamical ferromagnetic ordering). The result is the dynamical phase diagram reported in Fig. 7.

This figure shows that the dynamical ferromagnetic and paramagnetic phases $\mathrm{A}$ and $\mathrm{B}$ respectively, which touch each other at the dynamical critical point for $J=0$, withstand the effects of the quantum fluctuations intro- duced by having $J \neq 0$, apart from getting separated by a new phase $\mathrm{C}$ close to $g \simeq \bar{\lambda}$ (note the horizontal scale of Fig. 7). The robustness of phases $\mathrm{A}$ and $\mathrm{B}$ is further demonstrated in Fig. 8, which shows the timeevolution of the order parameter $\sigma^{x}(t)$ (first row) and of the spin wave density $\epsilon(t)$ (second row) within the dynamical ferromagnetic (first column) and paramagnetic (second column) phases, with $g / \bar{\lambda}=0.9$ and $g / \bar{\lambda}=1.5$ respectively. (Note that these values are well outside the range covered by Fig. 7.) The red solid and blue dashed lines correspond to increasing values of the coupling $J$ with spin waves, which, as anticipated, do not alter significantly the qualitative features of the dynamics. Note that in both the dynamical phases $\mathrm{A}$ and $\mathrm{B}, \epsilon(t)$ remains sufficiently small and therefore we expect the spin wave treatment developed in Sec. III to be accurate and these two phases to be robust.

Close to the mean-field dynamical transition point $g=\bar{\lambda}$, however, the system becomes extremely sensitive to non-equilibrium fluctuations, resulting in the peculiar phase C. In a typical point of this region, the dynamics of $\sigma^{x}(t)$ is driven by two processes, illustrated in the inset of Fig. 7: (a) the decay from a transient paramagnetic behavior to one of the two possible ferromagnetic sectors, and (b) the possible hopping between them. Heuristically, these phenomena occur when the energy of the macroscopic collective spin $\vec{\sigma}(t)$ is slightly above the barrier separating the two ferromagnetic minima. In this case the dynamical production of spin waves reduces the energy carried by $\vec{\sigma}$ and hence causes the dynamical trapping into one of the two ferromagnetic wells, accompanied by an increase of the spin wave density $\epsilon(t)$. The system is dynamically ferromagnetic, although it can occasionally hop to the opposite well, with a process assisted by the absorption of energy from the spin wave bath. The asymptotic sign of $\sigma^{x}(t)$, and therefore the sign of $\overline{\sigma^{x}}$, sensitively depends on the specific values of the parameters in a large portion of this novel dynamical ferromagnetic region ( $\mathrm{C}$ in Fig. 7), with a collective pseudo-aleatory character of the dynamics, which is illustrated in Fig. 9. Due to this sensitive dependence on the post-quench values of the parameters, which actually implies the same for the choice of the initial state, this phase C is referred to as "chaotic".

This dynamical behavior, obtained on the basis of the time-dependent spin wave theory, persists up to values $J / \bar{\lambda} \simeq 0.67$ of the coupling $J$, i.e., $J=2 \lambda$; at such strong coupling $\epsilon(t)$ grows significantly, invalidating the low-density spin-wave expansion. In order to explore this strong coupling regime, we relied on a time-dependent variational principle developed on matrix product states, see Sec. V.

The dynamics in the chaotic dynamical ferromagnetic region $\mathrm{C}$ may be understood qualitatively via a simple analogy: a coin toss. The toss corresponds to the sudden quench of the external field, where a macroscopic amount of energy is injected into the system in the form of regular macroscopic motion. The coin repeatedly hitting the 

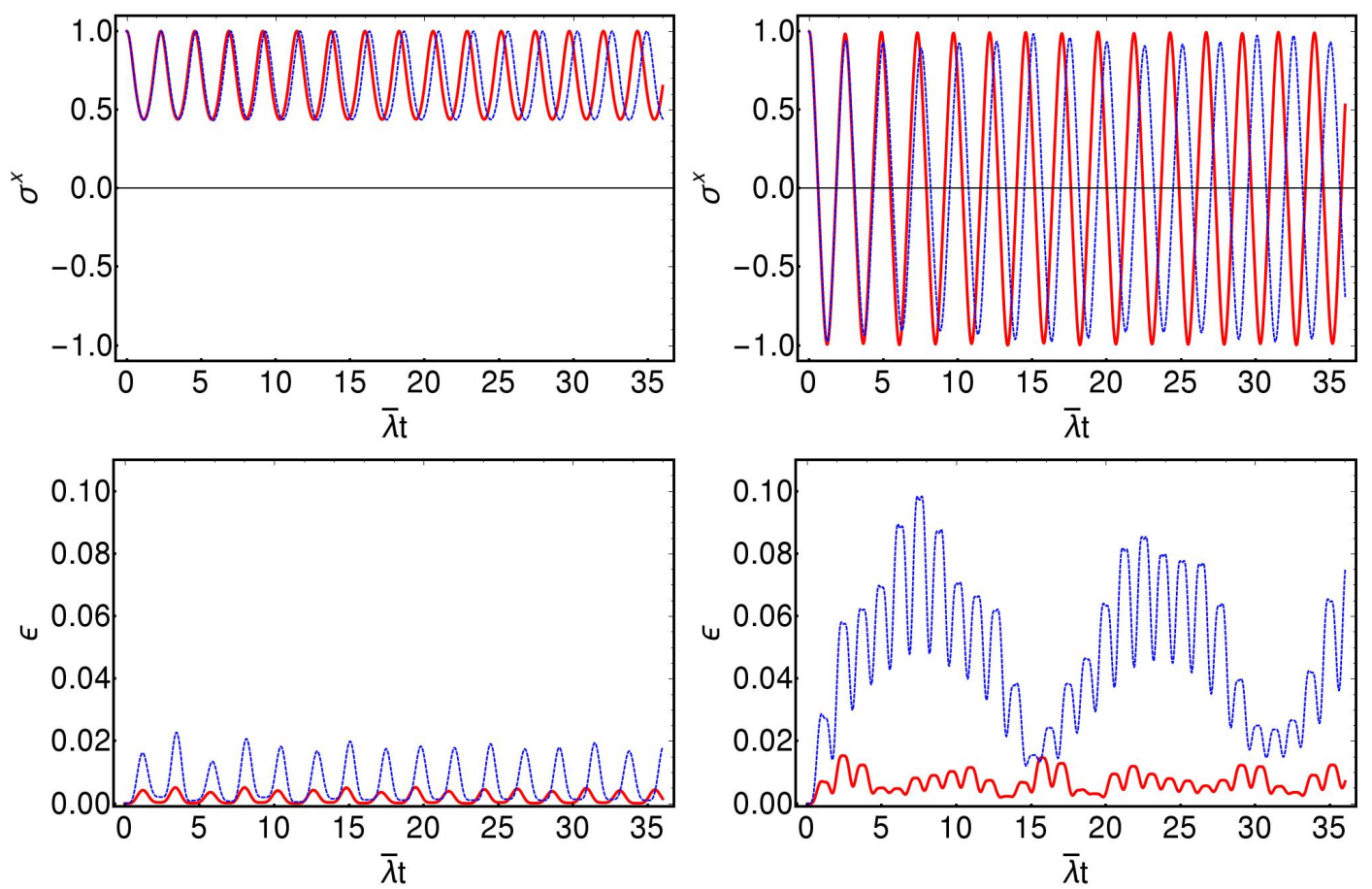

Figure 8. [Color online] Dynamical behavior of the order parameter $\sigma^{x}(t)$ (first row) and of the spin wave density $\epsilon(t)$ (second row) in the presence of a short-range interaction $J / \bar{\lambda}=0.1$ (solid red line) and 0.2 (dashed blue), after a quench from a fully polarized ferromagnetic state $\left(g_{0}=0\right)$. Left panels: dynamical ferromagnetic phase with $g / \bar{\lambda}=0.9$. Right panels: dynamical paramagnetic phase with $g / \bar{\lambda}=1.5$. These dynamical phases are characterized by the sign of the time-average of $\sigma^{x}(t)$, shown in the top panels. The quantity $\epsilon(t)$ shown in the bottom panels represents the total amount of spin wave excitations generated during the non-equilibrium evolution. This is the control parameter for the validity of the low-density expansion, which is consistent if $\epsilon \ll 1$, i.e., if the length of the total spin $|\vec{\sigma}(t)|=1-\epsilon(t)$ remains close to its maximal value. The presence of a short-range interaction, even of sizable strength $J / \bar{\lambda}=0.2$, produces a perturbative modification of the mean-field evolution and, correspondingly, a small amount of spin waves. In particular, the mean-field persistent oscillations are not damped by the self-generated "bath". In the plots, $N=100$ and the mean-field dynamical critical point is $g_{\text {dyn }} / \bar{\lambda}=1$.

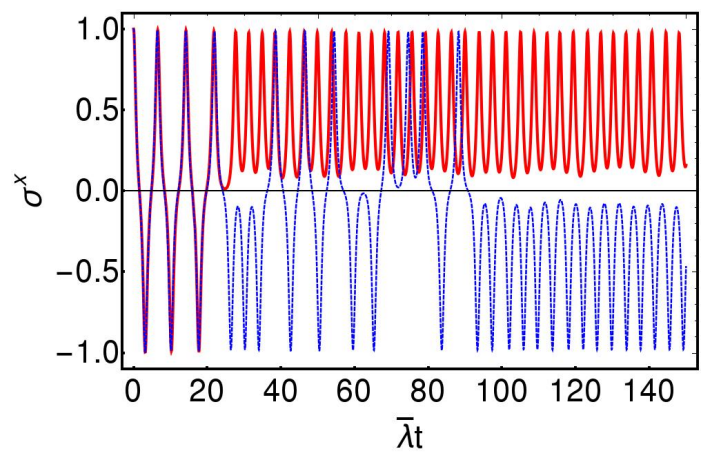

Figure 9. [Color online] Evolution of the order parameter $\sigma^{x}(t)$ within the chaotic dynamical ferromagnetic phase $\mathrm{C}$ in Fig. 7, for $g / \bar{\lambda}=1.03$ (solid red line) and 1.031 (dashed blue), and with $J / \bar{\lambda}=0.1$. Here $N=100$. The two lines are practically indistinguishable during the initial paramagnetic transient, but they have markedly distinct fates at the onset of the critical process denoted by (a) in the inset of Fig. 7 and they eventually end up into distinct wells. In both cases, $\epsilon(t)$ grows from $\epsilon(t=0)=0$ to values around 0.04 . This extreme sensitivity on the value of $g$ (and of $J$ ) is at the origin of the "mosaic" structure of region $\mathrm{C}$ in Fig. 7. ground and exciting its phonons corresponds to the loss of energy in favor of the microscopic degrees of freedom, i.e., the spin waves. Finally, the coin settling into one of the two macroscopically distinct stable configurations (heads or tails) corresponds to the trapping into one of the two ferromagnetic sectors $\left(\overline{\sigma^{x}}>0\right.$ or $\left.\overline{\sigma^{x}}<0\right)$. A diagram of the outcomes of the coin (heads or tails) as a function of the variables which parameterize the toss, would result in a picture very similar to region $\mathrm{C}$ of Fig. 7, as indeed shown in Ref. 67. Although the equations of motion in both cases are completely deterministic, the final outcome is extremely sensitive to the details of the dynamics and it can be considered as an effectively random process. We emphasize that we checked that the numerical results reported above in Fig. 7 and in the figures which follow are not affected as $N$ is increased (up to $N=400$ ).

\section{B. Generality of the chaotic dynamical phase}

We now show that the chaotic dynamical phase is not peculiar to the model in Eq. (61), but is actually expected 

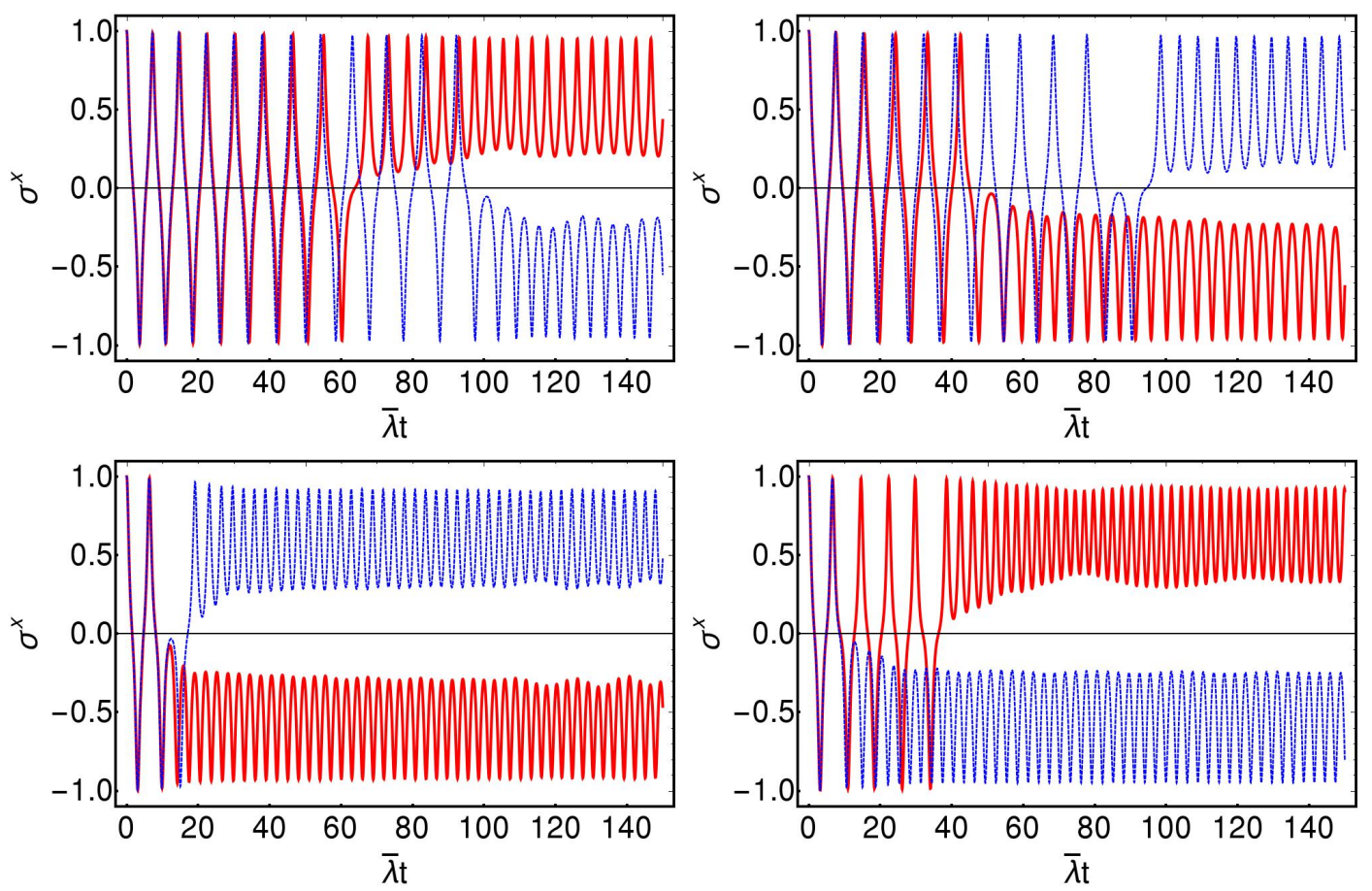

Figure 10. [Color online] Evolution of the order parameter $\sigma^{x}(t)$ after a quench from a pure ferromagnetic state $\left(g_{0}=0\right)$ in four different generalizations of the Ising Hamiltonian (20). Top left: XY spin chain with an infinite-range and a nearestneighbor interaction, defined by Eq. (63) with $\alpha_{y}=0.25, \alpha_{z}=0, g / \bar{\lambda}=1.03$ (solid red line) and 1.032 (dashed blue), with $J / \bar{\lambda}=0.4$. Top right: XYZ spin chain with an infinite-range and a nearest-neighbor interaction, defined by Eq. (63) with $\alpha_{y}=0.25, \alpha_{z}=0.125, g / \bar{\lambda}=0.9$ (solid red line) and 0.902 (dashed blue), with $J / \bar{\lambda}=0.4$. Bottom left: Ising spin chain with an infinite-range and a next-to-nearest-neighbor interaction, defined by Eq. (64) with $v(r)=\delta_{r, 1}+0.5 \delta_{r, 2}, g / \bar{\lambda}=1.03$ (solid red line) and 1.031 (dashed blue), with $J / \bar{\lambda}=0.2$. Bottom right: Ising spin chain with an infinite-range and a power-law decaying interaction, defined by Eq. (64) with $v(r)=1 / r^{2}, g / \bar{\lambda}=1.03$ (solid red) and 1.031 (dashed blue), with $J / \bar{\lambda}=0.2$. In all simulations, $N=100$. These trajectories have been obtained by numerically integrating the evolution equations given by the time-dependent spin wave theory, analogous to Eqs. (50), (54), derived for the generalized spin chains above through the same procedure as that explained in details in Sec. III B for the Ising model.

to emerge in a rather general class of ferromagnetic spin systems, characterized by competition between long- and short-range interactions.

First of all, the occurrence of the chaotic dynamical phase discussed in the previous section does not depend on the particular initial state we have chosen, as long as it has sufficiently strong magnetic ordering. In particular, the direction of the initial magnetization $\operatorname{Tr}[\rho(t=0) \vec{S}] \propto\left(\sin \theta_{0}, 0, \cos \theta_{0}\right)$ with $\theta_{0} \neq 0$ is immaterial, and the initial state $\rho(t=0)$ needs not be pure. This class encompasses all low-temperature equilibrium ordered states of $H\left(g<g_{\text {cr }}\right)$.

The chaotic dynamical phase occurs for arbitrary quantum spin magnitude, since a larger value of $s$ just amounts to rescaling the coupling strength $J$ in Eqs. (50), and therefore to decreasing the overall effect of the feedback from fluctuations on the evolution of the collective order parameter. In addition, in the limit $s \rightarrow \infty$, quantum fluctuations in the pre-quench ground state are suppressed as a consequence of the individual spins approaching their classical limit. Accordingly, the chaotic dynamical phase progressively disappears. However, thermal fluctuations can play a role similar to that of quantum fluctuations when initial states in equilibrium with a finite temperature $T>0$ are considered, leading to a non-vanishing feedback and thus to an extended chaotic dynamical phase even in the classical limit.

We also expect that the phenomena discussed here for a spin chain with an interaction characterized by $\mathbb{Z}_{2}$ symmetry should emerge also for other discrete symmetry groups. In the case of a "clock" symmetry $\mathbb{Z}_{n}$, for instance, the dynamical order parameter is expected to get eventually trapped into one of the $n$ distinct symmetrybreaking sectors, resulting in a multicolor version of the picture of Fig. 7 with different colors corresponding to the $n$ possible sectors. In this case, the appropriate heuristic analogy would be that of a "roulette" rather than a coin.

Furthermore, changing the short-range spin-spin interaction term $J$ from ferromagnetic to antiferromagnetic $(J<0)$ does not alter the structure of the phase diagram in Fig. 7. Indeed, the time-dependent spin wave theory evolution equations (50) do not change when $J \mapsto-J$, provided the substitution of the summation variable $k \mapsto \pi-k$ is performed ${ }^{83}$. 
We now turn our attention to generalizations of the Ising Hamiltonian (20). The top panel of Fig. 10 shows the evolution of the order parameter $\sigma^{x}(t)$ for the XY (top left panel) and XYZ (top right) versions of the LMG model with a nearest-neighbor anisotropic perturbation, defined by

$$
\begin{aligned}
& H_{X Y Z}=-\frac{\lambda}{N} \sum_{i, j=1}^{N}\left(\sigma_{i}^{x} \sigma_{j}^{x}+\alpha_{y} \sigma_{i}^{y} \sigma_{j}^{y}+\alpha_{z} \sigma_{i}^{z} \sigma_{j}^{z}\right) \\
& -g \sum_{i=1}^{N} \sigma_{i}^{z}-J \sum_{i=1}^{N}\left(\sigma_{i}^{x} \sigma_{i+1}^{x}+\alpha_{y} \sigma_{i}^{y} \sigma_{i+1}^{y}+\alpha_{z} \sigma_{i}^{z} \sigma_{i+1}^{z}\right) .
\end{aligned}
$$

with $\alpha_{z}=0$ (XY model) or non-vanishing values of $\alpha_{y, z}$ (XYZ model), while they reduce to the LMG model in Eq. (61) for $\alpha_{y}=\alpha_{z}=0$. Note that at the isotropic point $\alpha_{y}=1$ the discrete $\mathbb{Z}_{2}$ symmetry turns into a continuous $O(2)$ symmetry, as $\sigma^{z}$ is conserved. Consequently, the barrier separating the two ferromagnetic minima becomes increasingly shallow as this point is approached, which hinders the possibility for the collective order parameter to get trapped. Accordingly, the chaotic dynamical phase disappears in this limit.

The bottom panel of Fig. 10 shows the evolution of the order parameter $\sigma^{x}(t)$ for the LMG model with a next-tonearest-neighbor (bottom left) or algebraically decaying (bottom right) perturbation, defined by

$$
H_{L R}=-\frac{\lambda}{N} \sum_{i, j=1}^{N} \sigma_{i}^{x} \sigma_{j}^{x}-g \sum_{i=1}^{N} \sigma_{i}^{z}-J \sum_{i, r}^{N} v(r) \sigma_{i}^{x} \sigma_{i+r}^{x}
$$

where $v(r)$ decays to zero upon increasing the distance $r$ between the two interacting spins. For finite-range perturbations $v(r)$ has a compact support, while for powerlaw decaying interactions one has $v(r) \propto r^{-\alpha}$ with $\alpha>0$. The qualitative similarity of all the panels in Fig. 10 with the evolution displayed in Fig. 9 demonstrates that the chaotic behavior observed in the latter case is actually a generic phenomenon which emerges also in the generalized models discussed above. In particular, the evolution of a certain initial state under the effect of two close post-quench Hamiltonians (red and blue curves) results into two markedly different asymptotic states. Although Fig. 10 refers to specific choices of the various parameters involved, we verified that this strong sensitivity of the dynamics to the values of the parameters of the postquench Hamiltonian persists in a neighborhood of the points considered. We finally observe that the spatial dimensionality of the short-range perturbation does not play an important role, as well.

In summary, we have shown that the emergence of a chaotic dynamical phase is an ubiquitous phenomenon that requires essentially two sole physical ingredients, namely the spontaneous breaking of a discrete symmetry and a mean-field model perturbed by an interaction term with a non-trivial spatial dependence, which introduces fluctuations.

\section{Correlation function of the local order parameter}

According to the picture presented above, the prethermal dynamics of the system can be understood in terms of the motion of a classical, macroscopic degree of freedom (the collective spin $\vec{\sigma}$ ) coupled to a weakly interacting many-body system (the "bath" of spin waves), which, in turn, is driven by the former: see Eqs. (59) and (60). This driving mechanism is determined by the persistent precession of the collective spin and can be highlighted by studying the time- and space-dependent equal-time correlation functions $\left\langle\sigma_{j}^{x}(t) \sigma_{j+r}^{x}(t)\right\rangle$, of the local order parameter $\left\langle\sigma_{j}^{x}(t)\right\rangle$. Taking into account Eq. (25) at the leading order in the low-density expansion of Sec. III, the connected correlation function $C^{x x}(r, t)$ can be expressed as

$$
\begin{aligned}
C^{x x}(r, t) \equiv & \left\langle\sigma_{j}^{x}(t) \sigma_{j+r}^{x}(t)\right\rangle-\left\langle\sigma_{j}^{x}(t)\right\rangle\left\langle\sigma_{j+r}^{x}(t)\right\rangle \\
= & (\hat{X} \cdot \hat{x})^{2} \frac{1}{s}\left\langle q_{j}(t) q_{j+r}(t)\right\rangle+(\hat{Y} \cdot \hat{x})^{2} \frac{1}{s}\left\langle p_{j}(t) p_{j+r}(t)\right\rangle+2(\hat{X} \cdot \hat{x})(\hat{Y} \cdot \hat{x}) \frac{1}{s}\left\langle\frac{q_{j}(t) p_{j+r}(t)+p_{j}(t) q_{j+r}(t)}{2}\right\rangle \\
= & \cos ^{2} \theta(t) \cos ^{2} \phi(t) \frac{1}{N s} \sum_{k \neq 0} \cos (k r) \Delta_{k}^{q q}(t)+\sin ^{2} \phi(t) \frac{1}{N s} \sum_{k \neq 0} \cos (k r) \Delta_{k}^{p p}(t) \\
& -2 \cos \theta(t) \cos \phi(t) \sin \phi(t) \frac{1}{N s} \sum_{k \neq 0} \cos (k r) \Delta_{k}^{q p}(t) .
\end{aligned}
$$

Analogous expressions can be readily obtained for $C^{\alpha \beta}(r, t)$, with $\alpha, \beta=x, y, z$. In this section, for definite- ness, we focus on the perturbed LMG model of Eq. (61). 


\section{Modulated light-cone effect}

Density plots of the equal-time correlation functions $C^{x x}(r, t)$ are shown in Fig. 11. They are obtained by integrating the equations of motion (50) and (54) with the same initial conditions as in the previous sections and by substituting their solution $\left(\theta(t), \phi(t),\left\{\Delta_{k}^{q q}(t), \Delta_{k}^{q p}(t), \Delta_{k}^{p p}(t)\right\}\right)$ into Eq. (65), with $s=1 / 2$.

A light-cone effect ${ }^{84}$ is present for all values of the parameters $g$ and $J$, which is characterized by an exponentially fast decay in time of the correlation function for $|r|>2 v_{\text {max }} t$ with a certain $v_{\max }$, see Ref. 85. In fact, the infinite-range Hamiltonian generates a collective coherent precession of all the spins with no spatial structure, due to the full permutational symmetry of the spins. However, a non-trivial spatial dependence of the dynamical correlations arises in the presence of the additional shortrange interaction term, which results in a light-cone. A closer inspection of the figures reveals that a (seemingly) periodic modulation is superimposed to the amplitude of the correlations. The origin of this phenomenon can be explained in the following terms. Within the low-density expansion considered here, the quadratic bosonic Hamiltonian (30) governing the evolution of the spin waves has coefficients which depend parametrically on the angles $\theta(t), \phi(t)$. The latter evolve approximately periodically in time (cf. Fig. 8), resulting in an instantaneous dispersion relation of the spin waves with an approximately periodic time-dependence. The "stroboscopic" dynamics of the spin waves at integer multiples of the "period" of the collective precession can thus be argued to relax to a periodic or stroboscopic generalized Gibbs ensemble ${ }^{86,87}$, which is known to occur in quantum many-body systems subject to an external periodic driving. We emphasize, however, that here the periodic drive is given by the dynamics of the system itself, i.e., it is self-generated by the autonomous Hamiltonian dynamics, without external actions: the collective motion of the classical spin $\vec{\sigma}(t)$ generates an effective "external" drive for the spin waves, see Eq. (59).

Let us now investigate the behavior of the slope of the light-cone edge as a function of the system's parameters. This quantity can be derived in terms of the maximal velocity $v_{\max }$ of propagation of the quasi-particles, which can be computed as the maximal slope of their effective dispersion relation $\omega_{k}^{(\text {eff })}$ (see below). In the two limiting cases, $g \rightarrow 0$ and $g \rightarrow \infty$, this velocity $v_{\max }$ can be easily determined analytically.

For $g \ll \bar{\lambda}$, the classical spin performs small oscillations near the initial ferromagnetic configuration, hence $\theta(t) \approx$ $\pi / 2$ and $\phi(t) \approx 0$ (or $\pi$ ) for all times. The dispersion relation in such a near-equilibrium condition has already been determined in Eq. (40): in fact, the corresponding dispersion relation is asymptotically flat, $\omega_{k}^{(\text {eff })} \rightarrow 2 \bar{\lambda}$, as $g \rightarrow 0$, and hence $v_{\max }$ approaches zero in this limit. This is confirmed by numerical computations, as shown in the two top panels of Fig. 11 where the light-cone width shrinks as $g$ decreases.

In the opposite limit $g \gg \bar{\lambda}$, the collective spin approximately rotates uniformly along the equator, $\theta(t) \approx \pi / 2$, $\phi(t) \approx 2 g t$, at frequency $2 g$. The effective (Floquet) Hamiltonian ${ }^{88}$ of the spin waves is simply given by the time-averaged Hamiltonian to lowest order in the driving period $\pi / g$. Thus, by averaging in time the coefficients of Eq. (59), we find the effective dispersion relation

$$
\omega_{k}^{(\mathrm{eff})}=4 \sqrt{\bar{\lambda}(\bar{\lambda}-J \cos k)}
$$

and therefore, for small $J / \bar{\lambda}$, the maximal velocity of propagation is given by

$$
v_{\max }=\max _{k}\left|\frac{\partial \omega_{k}^{(\mathrm{eff})}}{\partial k}\right| \sim J
$$

Comparing this prediction with the slope $2 v_{\max }$ of the light-cone of correlations, we find fairly good agreement with the data shown in the bottom left panel of Fig. 11.

A more precise quantitative determination of the lightcone edge from numerics requires some care. In order to address this, consider a quantum system composed by free quasi-particles with dispersion relation $\omega_{k}$, and assume parity symmetry, i.e., $\omega_{k}=\omega_{-k}$. An equaltime, two-point correlation function can be generically expressed as (see, e.g., Ref. 69)

$$
C(r, t)=\int_{-\pi}^{\pi} \frac{d k}{2 \pi} f(k) e^{i k r-i 2 \omega_{k} t}
$$

where the function $f$ depends on the model and on the quench. In the scaling limit of large $r$ and $t$ with fixed $r / t \equiv \xi, t \rightarrow \infty$, this correlation function shows a different asymptotic behavior along rays within or outside the causal region delimited by the light cone $|\xi| \leq 2 v_{\max }$. Indeed, one finds

$$
C(\xi t, t) \underset{t \rightarrow \infty}{\sim} \begin{cases}\sum_{k^{*}} f\left(k^{*}(\xi)\right) \frac{\exp \left[i\left(k^{*}(\xi) \xi-2 \omega_{k^{*}}(\xi)\right) t\right]}{\sqrt{2 \pi \omega_{k^{*}(\xi)}^{\prime \prime}}}, & \text { for }|\xi|<2 v_{\max } \\ A \exp (-\delta(\xi) t), & \text { for }|\xi|>2 v_{\max }\end{cases}
$$

where $k^{*}(\xi)$ is a solution to the equation $2 \partial \omega_{k} / \partial k(k)=\xi$, which exists only if $|\xi|<2 v_{\max }$, and the sum runs over 

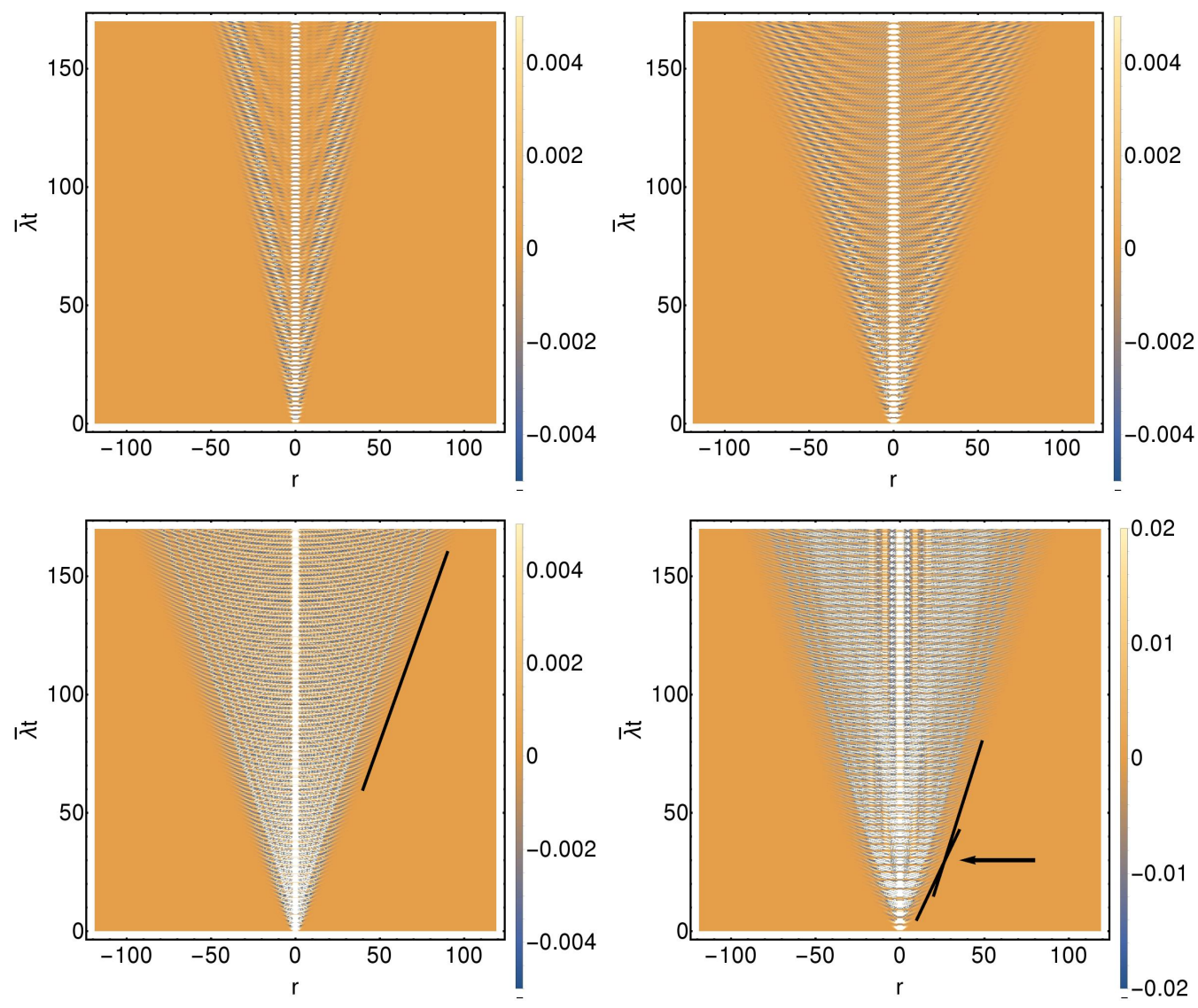

Figure 11. [Color online] Space-time density plots of the dynamical correlation function $C^{x x}(r, t)$ [see Eq. (65)] after a quench of the magnetic field $g$ from a fully polarized ferromagnetic state $\left(g_{0}=0\right)$ to $g / \bar{\lambda}=0.7,0.9,1.025,3$, in clockwise order from top left. In all plots, $J / \bar{\lambda}=0.25, N=240$. For a small quench occurring deep in the ferromagnetic phase (top left), the overall amplitude of the correlation function is weak (few excitations are produced) and the light-cone is narrow due to an almost constant spin waves dispersion relation. The amplitude and the width become larger as the dynamical critical region is approached (top right). In the chaotic dynamical phase (bottom right), a "knee" is visible, marked by the black arrow, witnessing a change of the maximal velocity of propagation due to the trapping of the orbit, after a paramagnetic transient, into a ferromagnetic sector (notice the change of scale, highlighting a larger amplitude of the correlations). Finally, deep in the paramagnetic phase (bottom left), the maximal velocity approaches the value analytically predicted in Eq. (67), indicated by the black line. An approximately periodic modulation of the amplitude of $C^{x x}(r, t)$ is visible in all cases, which reflects the approximately periodically driven nature of the spin waves, induced by the precession of the collective spin.

the set of such solutions. Accordingly, upon increasing the time $t$, the correlation function decays to zero as $t^{-1 / 2}$ along rays within the light-cone, whereas it decreases exponentially along rays outside the light-cone (the latter is a general fact valid for all systems with short-range interactions, as follows from the Lieb-Robinson bound ${ }^{85}$ ). The proper way of extracting $v_{\max }$, and thus of defining the light-cone edge from the numerical data, is therefore by inspecting the decay of the correlation function along space-time rays and thereby discriminating powerlaw from exponential decay: the critical ray which separates the two behaviors is the light-cone edge, and its slope unambiguously determines the maximal velocity of propagation of excitations within the system. Figure 12 shows that the two scaling behaviors in Eq. (69) are indeed found in the the numerical data. This agrees with the picture of self-consistently periodically driven spin waves.

\section{Dynamical correlations in the chaotic dynamical phase}

The chaotic dynamical ferromagnetic phase $\mathrm{C}$ in Fig. 7 leaves detectable signatures on the dynamics of the local order parameter correlation functions.

The self-consistent internal driving provided by the collective spin dynamics changes when the transient paramagnetic behavior turns into an evolution occurring eventually within one of the ferromagnetic sectors, as happens, e.g., in Fig 9. From the point of view of the spin waves, this can be seen as a change of their effective (Floquet) Hamiltonian, which, accordingly, results in a change of the associated "speed of light". Although the 

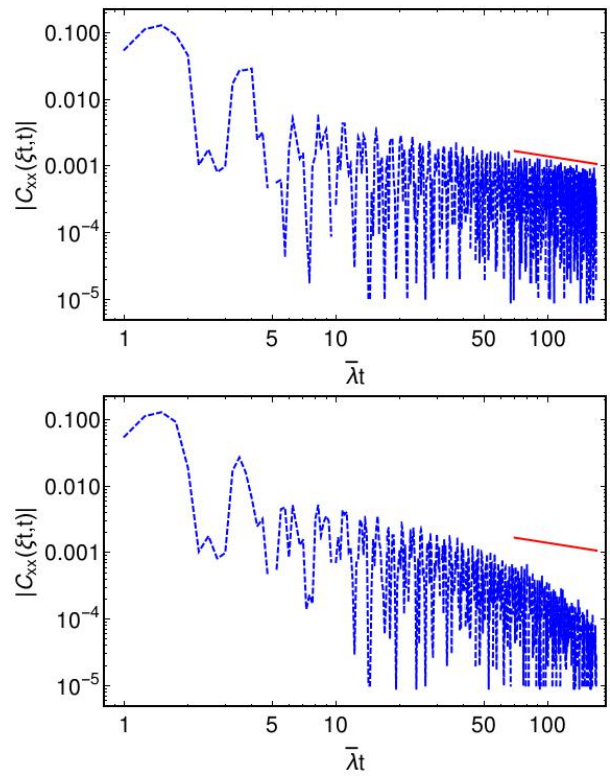

Figure 12. [Color online] Long-time behavior of the correlation function $C(\xi t, t)$ along two close spacetime rays with fixed $\xi=0.5 \bar{\lambda}$ (left) and $0.55 \bar{\lambda}$ (right) in a log-log scale, after a quench of the magnetic field from a pure ferromagnetic state $\left(g_{0}=0\right)$ to $g / \bar{\lambda}=0.9$ with $J / \bar{\lambda}=0.25, N=240$, corresponding to the data of the top right panel of Fig. 11. Apart from an (approximately) periodic modulation, $C(\xi t, t)$ decays, in the large spacetime limit, as a power-law (left) or as an exponential (right) as a function of time. The red line highlights the $t^{-1 / 2}$ decay suggested by the argument in the text, see Eq. (69). Data are consistent with a maximal velocity of propagation of the effective free quasiparticles between $0.25 \bar{\lambda}$ and $0.275 \bar{\lambda}$ in this specific numerical instance.

values of $v_{\max }$ before and after this change from dynamical paramagnet to dynamical ferromagnet, are not very different, a variation of slope in the light-cone is visible in some of the numerical computations, e.g., those reported in the bottom right panel of Fig. 11. They correspond to the macroscopic, qualitative change in the internal driving provided by $\vec{\sigma}(t)$. This phenomenon is a consequence of the existence of a chaotic dynamical ferromagnetic phase, and it can be seen as a further, characteristic hallmark of its peculiar nature.

\section{Ramp dynamics}

We now extend the analysis of the previous Section and of Ref. 66 for the system described by the Hamiltonian (20) to a time-dependent ramp $g(t)$ of the transverse field, describing the crossover from the sudden quench (infinitely quick ramp) to the adiabatic evolution (infinitely slow ramp). We consider a linear time- dependence

$$
g(t)= \begin{cases}g_{0}, & \text { for } t<0 \\ g_{0}+\left(g-g_{0}\right) \frac{t}{\tau}, & \text { for } 0 \leq t \leq \tau \\ g, & \text { for } t>\tau\end{cases}
$$

The parameter $\tau$ controls the total duration of the ramp. The system is initialized in the ground state of $H\left(g_{0}\right)$ at $t_{0}<0$ and then evolves with the time-dependent Hamiltonian $H(g(t)))$ at later times. When $\bar{\lambda} \tau \ll 1$, the results approach those obtained for the quantum quench dynamics of the previous section, see Fig. 7. Upon increasing $\tau$, we expect two effects. (i) First, the final state for $t \geq \tau$, will be progressively closer to the adiabatic one (i.e., the ground state of the final Hamiltonian, since the system is initialized in a zero-temperature ground state); this implies that the dynamical critical point, separating non-equilibrium trajectories within one ferromagnetic well from the dynamically paramagnetic ones encircling both wells, will move towards the equilibrium critical point, which is asymptotically reached in the adiabatic limit $\tau \rightarrow \infty$. This phenomenon occurs also in the absence of fluctuations, i.e., within the LMG model. (ii) Second, in the presence of fluctuations, an increasingly slower protocol will deposit in the system a progressively smaller amount of energy in the form of spin wave excitations with $k \neq 0$. By inspecting the Hamiltonian (59) or the equations of motion, one notices that the driving $g(t)$ directly affects only the dynamics of the collective spin. This macroscopic precession, in turn, causes the production of pairs of spin waves with opposite quasi-momenta: Near the dynamical transition, the self-generated bath of spin waves dissipates the energy of the classical spin, causing its trapping into either of the ferromagnetic wells. Accordingly, the smaller the amount of spin waves, the smaller the region of the parameter space within which the trapping phenomenon can occur is, and we therefore expect that the chaotic dynamical ferromagnetic phase $\mathrm{C}$ will shrink as $\tau$ increases.

This picture is confirmed by the numerical integration of the equations of motion, as one can see from Figs. 13 and 14. In particular, Fig. 13 shows how the dynamical phase diagram in Fig. 7 changes upon increasing, from left to right, the duration of the ramp $\tau$ in $g(t)$ which takes it from the initial value $g_{0}$ to the final value g. As expected, the chaotic region $\mathrm{C}$ in the parameter space shrinks with its two boundaries getting increasingly closer to each other, while region $\mathrm{C}$ as a whole moves towards the line at which the transition occurs in equilibrium, see Eq. (43).

In order to highlight this shift and the fate of the chaotic phase, the left panel of Fig. 14 shows a cut of the phase diagrams in Fig. 13 corresponding to a fixed value $J / \bar{\lambda}=0.2$ along the horizontal axis, and how the corresponding phases as a function of $g / \bar{\lambda}$ change as $\bar{\lambda} \tau$ increases well beyond the values considered in Fig. 13. In order to assess the reliability of the spin wave approximation on which our analysis rely, the right panel of Fig. 14 
shows with colorcode how fast the long-time averaged spin wave density $\epsilon(t)$ [see Eq. (52)] decreases upon increasing the ramp duration and as a function of $g / \bar{\lambda}$ for the same conditions as in the left panel.

\section{STRONG INTERACTIONS}

In order to check the robustness of the observed phenomena in the presence of a nearest-neighbor interaction strength $J$ increased beyond the perturbative regime considered in the previous sections, we simulate numerically the evolution of the system by using the time-dependent variational principle developed in Refs. 89 and 90. This formulation requires a matrix product operator (MPO) representation of the Hamiltonian. Since the interaction strength of the infinite-range part of the Hamiltonian (20) scales with the system size $N$ it is not possible to rewrite the thermodynamic limit of the Hamiltonian in the MPO form and hence to simulate the time evolution directly in the thermodynamic limit. Accordingly, we performed finite-size simulations on long chains, up to $N=400$.

The reformulation of the Hamiltonian in the MPO form is done in two steps. First, we write an exact homogeneous MPO with a large bond dimension $D_{\mathrm{MPO}}=$ $N+1$ and then use standard methods in order to find a compact inhomogeneous MPO representation with a bond dimension up to $D_{\mathrm{MPO}}=17$ and an error below $10^{-10}$. In all simulations we used a time step of 0.02 (in units of $\lambda$ ), a matrix product state bond dimension up to $D=600$, and the second order single-site integrator proposed in Refs. 89 and 90.

\section{A. The trajectories and the phase diagram at large nearest-neighbor interactions}

We first verify that the sensitivity of the evolution and of the final state to the values of the quench parameters observed in the perturbative regime of small spin wave density $\epsilon$ carries over to a larger nearest-neighbor interaction strength $J$. By extensive numerical simulations we show that indeed both phenomena persist as summarized in Fig. 15, where we show the dependence of the long-time time-averaged value of the order parameter $\overline{\sigma^{x}}$ as a function of the transverse field $g$ for various values of $J$ around $0.5 \bar{\lambda}$, i.e., $J \approx \lambda$. In particular, depending on the value of $J$, the dynamical ferromagnetic phase with $\overline{\sigma^{x}}>0$ at $g / \bar{\lambda} \lesssim 0.9$ turns into a dynamical paramagnetic phase with $\overline{\sigma^{x}}=0$ at $g / \bar{\lambda} \gtrsim 1.3$ via an intermediate region in which the ferromagnetic ordering is reversed as compared to the initial one, i.e., $\overline{\sigma^{x}}<0$. This is reminiscent of the "stripes" of reversed magnetization in Fig. 7 in the leftmost part of region $\mathrm{C}$. We therefore expect a "chaotic" region to be present in between.

Far from the mean-field dynamical critical point $g_{\mathrm{dyn}}=$ $\bar{\lambda}$, the time evolution of the order parameter remains qualitatively similar to the mean-field case. As shown in
Fig. 16 , for $g / \bar{\lambda}=0.5$ and $g / \bar{\lambda}=1.5$ the ferromagnetic (red solid line) and paramagnetic (blue solid) trajectories are only slightly shifted with respect to the mean-field evolution ( $J=0$, dashed red and blue lines) despite the large interaction strength $J=0.5 \bar{\lambda}=\lambda$.

Upon getting closer to the mean-field dynamical critical point $g=\bar{\lambda}$ with $J \neq 0$ one observes, instead, significant qualitative changes in the time evolution of $\sigma^{x}(t)$ compared to the case $J=0$, as shown in Figs. 17 and 18. In particular, in the region of parameters highlighted in Fig. 15 one observes that the eventual sign of $\sigma^{x}(t)$ is reversed compared to the initial value and that it is attained possibly after a number of sign changes, as in Fig. 17. This final sign reversal appears to be stable in longer simulations. The dynamics, however, become more complex when $g$ approaches the dynamical paramagnetic phase: as expected, the associated instability significantly affects the resulting evolution of $\sigma^{x}(t)$ which is displayed in Fig. 18 and which is characterized by a sensitive dependence of the long-time magnetization on the quench parameter (magnetic field). Correspondingly, the time evolution of the order parameter looks irregular before it settles in one of the two sectors with a definite sign of $\sigma^{x}$. For some trajectories visible in both Figs. 17 and 18, the order parameter oscillates between them before it eventually reaches the final magnetization sector. In this case, the period of these oscillations progressively increases before the "trapping" occurs. Similarly to the case of the propagation of correlations at weak interactions discussed in Sec. IV C, this change of the oscillation frequency corresponds to a transition from a dynamically paramagnetic to a dynamically ferromagnetic regime. Trajectories of Fig. 18 are marked in the phase diagram shown in Fig. 15 by black crosses.

In summary, the qualitative picture of the phases observed at small interactions persists also at large $J$. We emphasize the fact that for the values of $J$ used in the simulations reported in this Section, the accuracy of the spin-wave approach is poor and no quantitative agreement between the two methods has to be expected. In turn, at smaller values of $J$ and for the largest system sizes $N$ reached in these simulations, the time scale over which the collective magnetization gets trapped into a ferromagnetic sector is larger than the Ehrenfest time scale $T_{\mathrm{Eh}} \lesssim \mathcal{O}(\sqrt{N})$ over which the motion of the collective magnetization is approximately classical (see Secs. II C and VI). This fact makes it difficult to observe the chaotic dynamical phase in this regime with MPS-TDVP. For this reason, the two methods used in this paper effectively explore complementary regimes of the dynamics of the system and they cannot be quantitatively compared.

\section{B. Correspondence between the zeros of the order parameter and the cusps of the return probability}

In addition to the dynamical phase transition in the qualitative features of the long-time behavior of the or- 

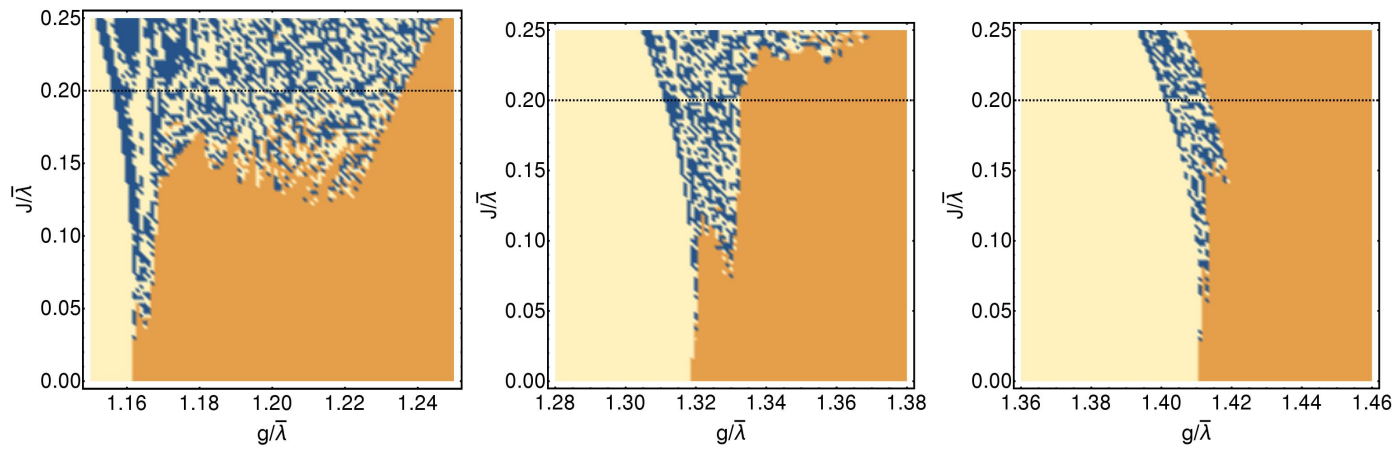

Figure 13. [Color online] Dynamical phase diagrams for linear ramps [see Eq. (70)] of the transverse magnetic field for the model described by Eq. (20) starting from $g_{0}=0$ (fully polarized ferromagnetic state), in the plane of the dimensionless final magnetic field $g / \bar{\lambda}$ and short-range interaction strength $J / \bar{\lambda}$, analogous to Fig. 7 . Here $N=100$. The color of each point in the diagrams indicates the asymptotic sign of the time-averaged order parameter, with the same graphical conventions as in Fig. 7. The dimensionless duration $\bar{\lambda} \tau$ of the ramp is 0.7 (left), 1.00 (middle), 1.15 (right). As the driving becomes slower, the mean-field dynamical critical point for $J \rightarrow 0$ shifts from the sudden quench value $g_{\text {dyn }} / \lambda=1$ towards that in the adiabatic limit, i.e., the equilibrium critical point $g_{\mathrm{cr}} / \lambda=2$ which is witnessed by the progressive shift rightwards along the horizontal axis of the border between the yellow and orange regions in the plot. Simultaneously, the chaotic dynamical ferromagnetic phase shrinks, due to the progressively smaller amount of non-equilibrium excitations produced by the increasingly slower ramp.
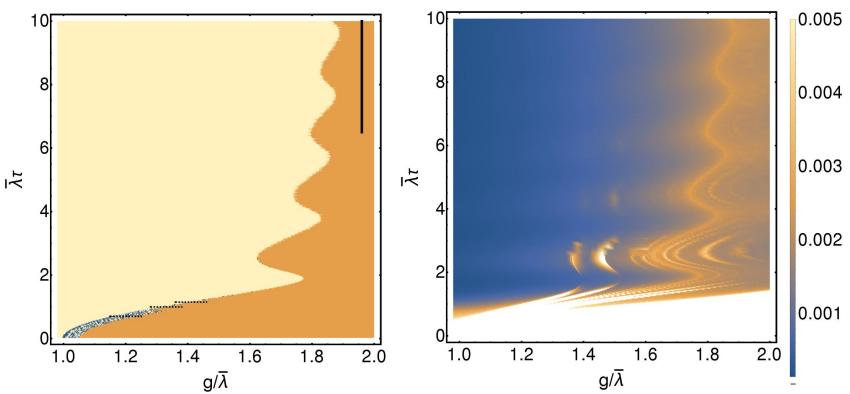

Figure 14. [Color online] Left panel: Dynamical phase diagram for linear ramps of the magnetic field $g$ of the same model as in Fig. 13, in the plane of the dimensionless final magnetic field $g / \bar{\lambda}$ and of the dimensionless ramp duration $\bar{\lambda} \tau$, but with fixed $J / \bar{\lambda}=0.2$. The color of each point of the diagram is assigned as in Figs. 13 or 7 , and the diagram corresponds to taking a horizontal cut of those in Fig. 13 at fixed $J / \bar{\lambda}=0.2$ and varying $\tau$ continuously. As the ramp becomes slower, we notice two features: first, the two boundaries of the chaotic phase shift from the sudden quench position around $g_{\mathrm{dyn}} / \bar{\lambda}=1$ towards the equilibrium critical point $g_{\text {cr }} / \bar{\lambda}=2-(5 / 8)(J / \bar{\lambda})^{2}+\mathcal{O}(J / \bar{\lambda})^{3} \approx 1.975$ [see Eq. (43)] in the adiabatic limit, marked by the black vertical line. Second, the chaotic dynamical phase shrinks and practically disappears as $\tau$ is increased. Both these features are clearly visible in the picture. The "oscillatory" dependence of the phase boundary on $\tau$ is already present at the mean-field level. Right panel: long-time average of the density $\epsilon(t)$ of spin wave excitations generated in the non-equilibrium dynamics.

der parameter discussed so far, another type of dynamical criticality related to non-analytic behavior of the return probability to the ground state manifold has been proposed in Refs. 61 and 91, as discussed in the Introduction. In Ref. 63 an intimate connection between them has been observed by studying a transverse field Ising model
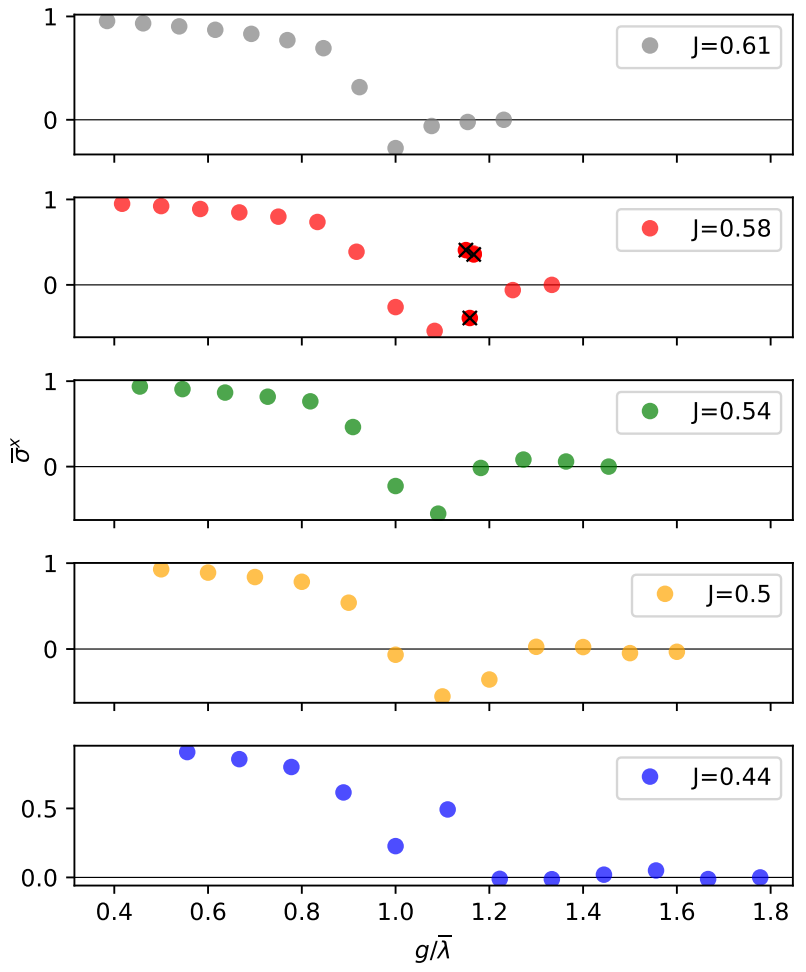

Figure 15. [Color online] Time-averaged order parameter $\overline{\sigma^{x}}$ of the model of Eq. (61) as a function of the post-quench values $g$ of the transverse field, for various values of the coupling $J$ around 0.5 , with $\bar{\lambda}=\lambda+J=1$. The black crosses $(J=0.58)$ correspond to a finer grid of values of $g$, with $\delta g=0.008$ are shown in order to display the high sensitivity of the chaotic phase to post-quench parameters. These data show that the dynamically ferromagnetic and chaotic region persist also at large nearest-neighbor interactions. The data is calculated for system size $N=200$. 


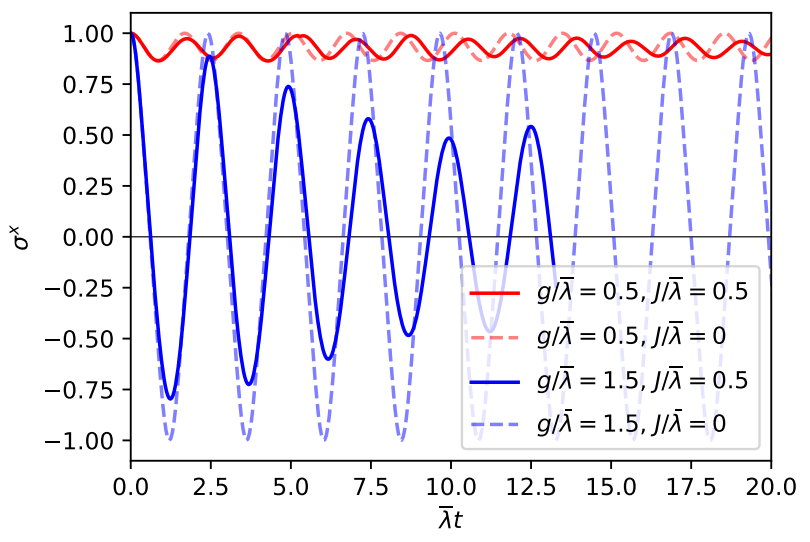

Figure 16. [Color online] Comparison between the evolution of the order parameter $\sigma^{x}$ at large $J$ (solid lines) with those of the mean-field model with $J=0$ (dashed lines) far from the critical region and for the same model as in Fig. 15. We observe that the evolution corresponding to both the ferromagnetic and paramagnetic phases are not altered qualitatively by the effects of quantum fluctuations. The decay of the oscillations amplitude upon increasing time is a finite-size effect. In these simulations $N=400$ and $D=300$.

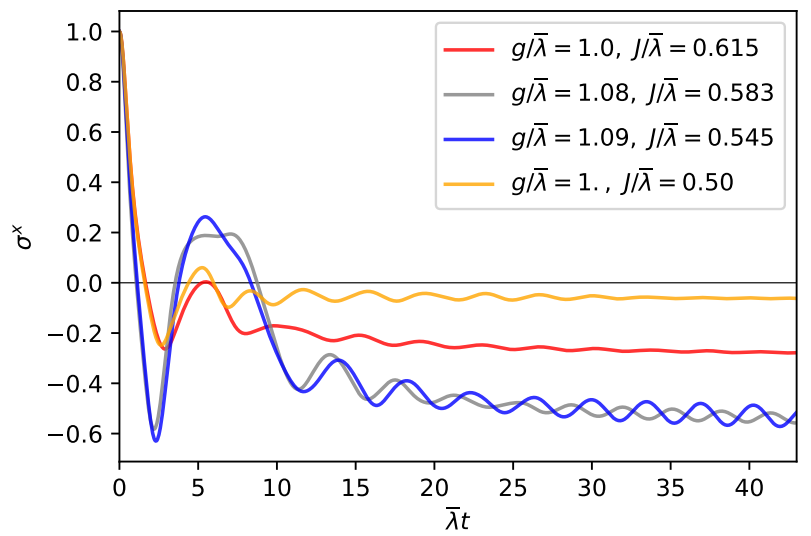

Figure 17. [Color online] Stability of the flipped ferromagnetic region. We show several trajectories within a wide range of different quench parameters as a part of the same region with a flipped final magnetization. This demonstrates stability of the flipped ferromagnetic region at large nearest-neighbor interactions. Simulations were performed with $N=200, D=200$.

with variable-range interactions similar to that discussed in this work. We provide here numerical evidence that this correspondence remains valid also when the meanfield infinite-range Hamiltonian is perturbed by nearestneighbor interactions studied in this work. In particular we demonstrate that the cusps in the time evolution of the return probability $P(t)$ to the ground state manifold are simultaneous with the zeros of the time-dependent order parameter $\sigma^{x}(t)$, since $P(t)=P_{1}(t)+P_{2}(t)$, where $P_{1}(t)$ is the probability to return to the initial state, and $P_{2}(t)$ is the probability to end up in the state with opposite longitudinal magnetization, cusp singularities are

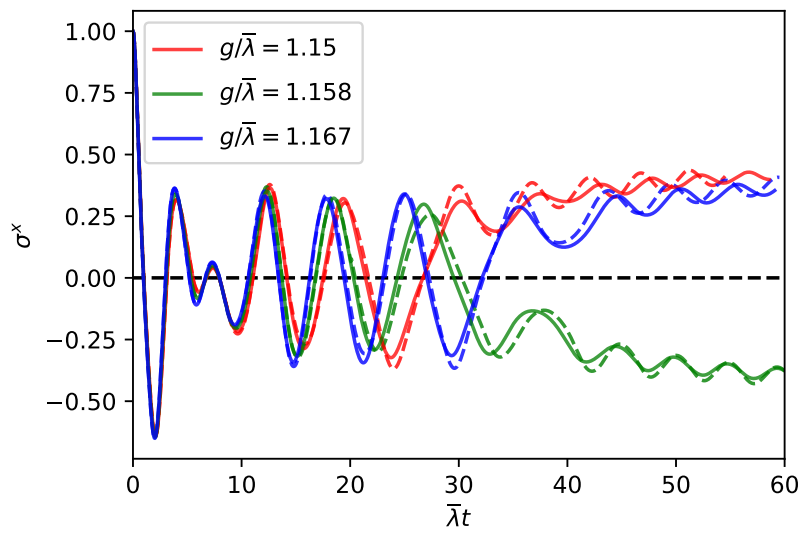

Figure 18. [Color online] Evolution of the order parameter $\sigma^{x}(t)$ for a quench occurring close to the mean-field dynamical critical point within the chaotic phase. These curves at fixed $J / \bar{\lambda}=0.583$ show a sensitive dependence on the value of $g$, and they may oscillate for a long time before settling eventually in a sector with definite positive or negative order parameter. By changing the quench parameter $g / \bar{\lambda}$ only slightly (approximately by 0.08 ) we observe a large change in the final magnetization which jumps from the positive to the negative sector and finally back to the positive sector. The curves in this plot correspond to the data points indicated by black crosses in Fig. 15. Simulations were performed with $N=200$, and with $D=600$ (full lines) and $D=500$ (dashed lines).

expected whenever $P_{1}(t)=P_{2}(t)$. Notice that both $P_{1}$ and $P_{2}$ become dramatically small as a function of time as a result of the excitation of spin waves.

Deep in the ferromagnetic region, shown in Fig. 19, the order parameter $\sigma^{x}(t)$ remains positive, and, in fact, we observe that the probability $P_{1}$ to return to the initial state is always much larger than the probability $P_{2}$ to reach the state with an opposite magnetization. This region thus corresponds to a non-vanishing order parameter and the absence of cusps in the return probability.

By increasing the magnetic field $g$ we enter a region where the order parameter $\sigma^{x}$ vanishes at certain times but later remains finite for a long time. Also in this region we observe that the zeros of the order parameter are close to the cusps in the return probability as shown in Fig. 20. Similarly the reversal of the final magnetization of one of the trajectories (left panels) corresponds to a larger probability $P_{2}$, which in this case becomes much larger than the probability to return to the initial state $P_{1}$. The correspondence remains valid also in the paramagnetic region, as shown in Fig. 19.

Finally, we remark that the convergence properties with respect to the bond dimension are better in the ferromagnetic and paramagnetic regions than in the chaotic region. Far from the critical point the simulations for the system size $N=400$ converged already with bond dimension $D=300$. On the other hand, in the chaotic region we needed bond dimensions around $D=600$. Therefore, 

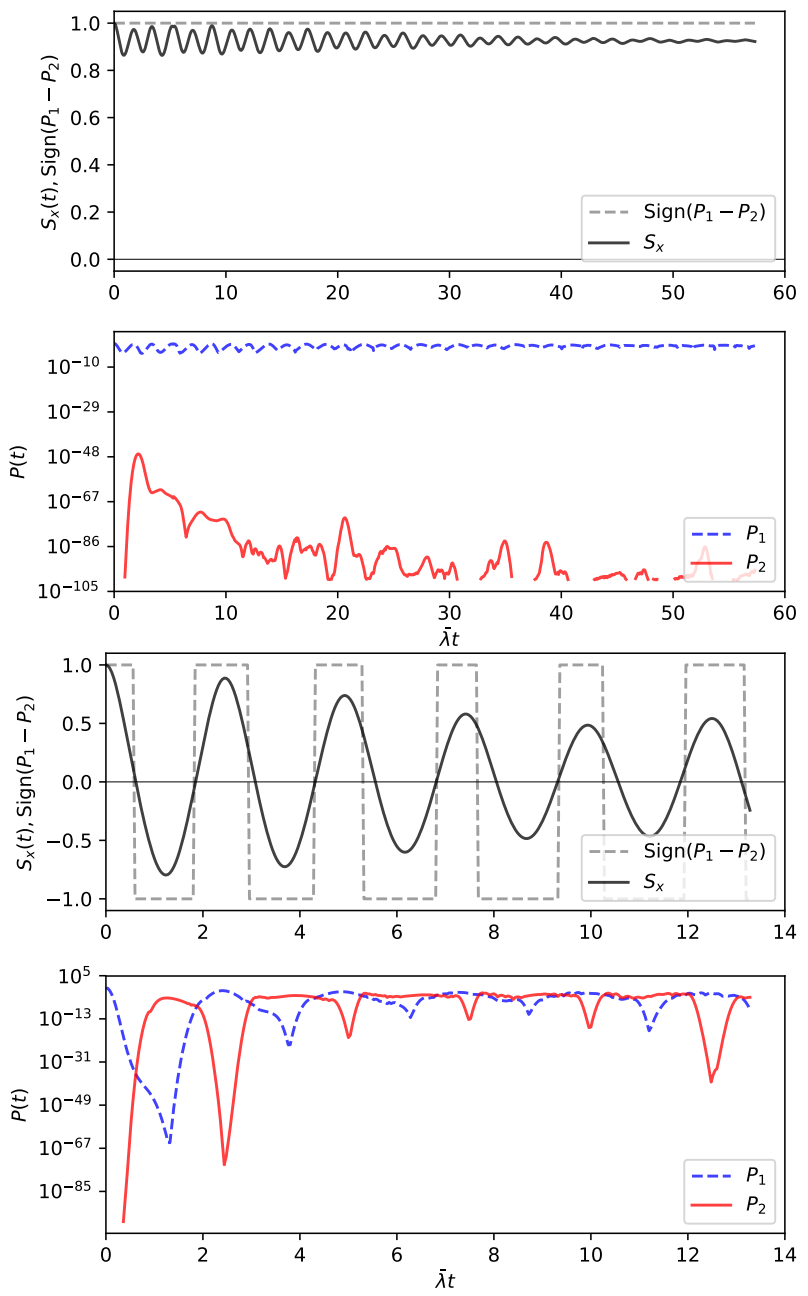

Figure 19. [Color online] Relationship between the vanishing of the order parameter $\sigma^{x}$ and the change of sign of the difference between the probability $P_{1}$ to return to the initial state and the probability $P_{2}$ to reach the state with the opposite magnetization. Upper panels: comparison between the order parameter (orange) and the sign of $\left(P_{1}-P_{2}\right)$ (blue). Lower panels: evolution of the return probabilities $P_{1}$ (blue) and $P_{2}$ (red). In the ferromagnetic region (left) we observe that the order parameter remains close to one, which corresponds to a large difference in the probabilities $P_{1}$ and $P_{2}$. The return probability to the initial state, $P_{1}$, remains at all times much larger than the return probability to a state with the opposite magnetization, $P_{2}$. On the other hand, in the paramagnetic region (right panels) the oder parameter periodically changes the sign. These changes correspond well with the cusps in the return probability which appear at points where $P_{1}-P_{2}$ changes its sign. Parameters: $D=300, N=400, J / \bar{\lambda}=0.5$, $g / \bar{\lambda}=0.5$ (top), 1.5 (bottom).

we could perform simulations only up to the system size $N=200$. In addition, the probabilities $P_{1}$ and $P_{2}$ are closer in the chaotic region, where the system initially oscillates between the positive and negative magnetization sector and only later remains in one or the other. Hence, the finite-size effects are noticeably larger in this
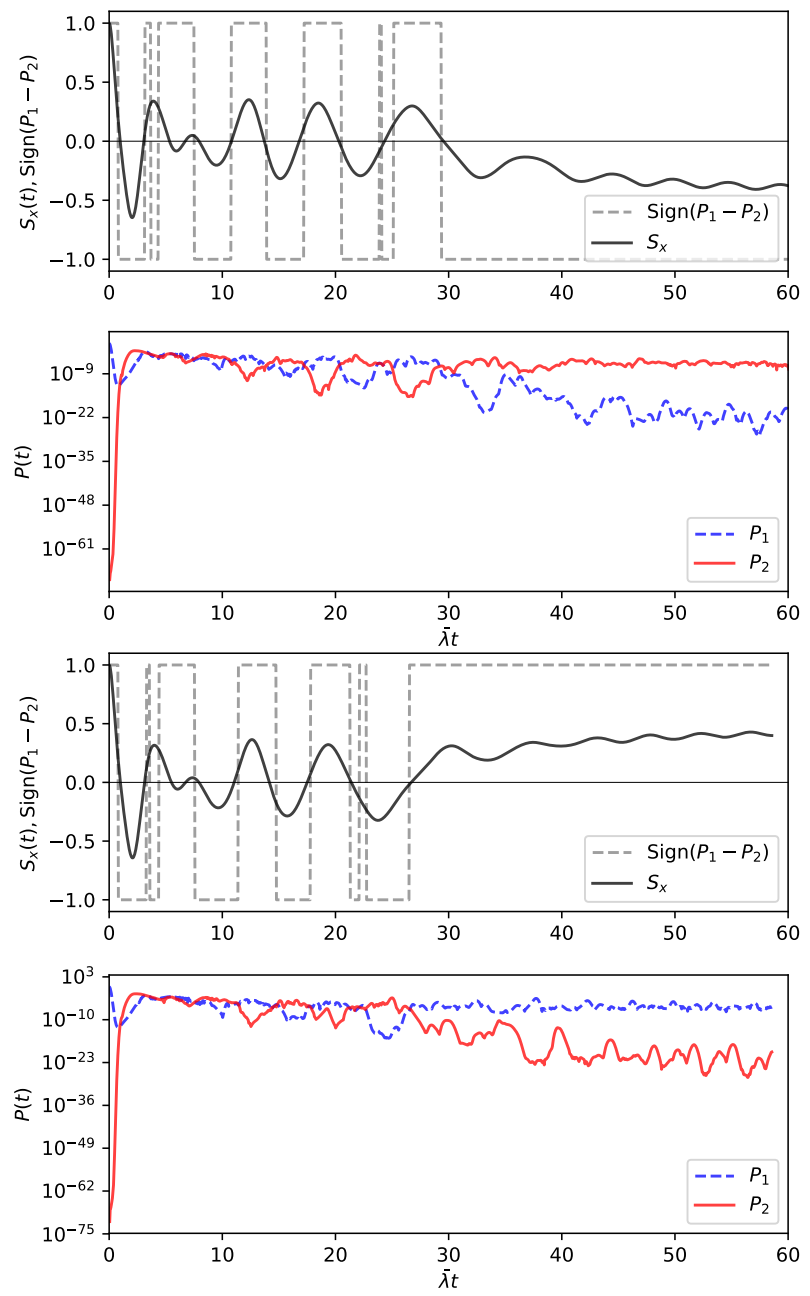

Figure 20. [Color online] Same plots as in Fig. 19 with different values of the parameters corresponding to the flipped and chaotic ferromagnetic regions. Initially the zeros of the order parameter correspond precisely to the cusps in the return probability, i.e., to $P_{1}=P_{2}$. At intermediate times the quality of this correspondence decreases, due to finite-size effects (and is improved by increasing the system size). (left) At late times the return probability $P_{2}$ to the state with the opposite magnetization, becomes larger than the return probability $P_{1}$ to the initial state. This corresponds well to the flipped time-dependent order parameter at late times. (right) By changing the parameters only slightly we the final magnetization changes its sign, but the correspondence between the zeros of the order parameter and the cusps in the return probability remains valid. Parameters: $D=600, N=200$, $J / \bar{\lambda}=0.583, g / \bar{\lambda}=1.15$ (top), 1.158 (bottom).

region. We, however checked that the zeros of the order parameter and the cusps in the return probability move closer as the system size is increased. Despite relatively large finite-size effects in the chaotic region we observe a clear tendency that $P_{1}>P_{2}$ when $\sigma^{x}>0$ and $P_{1}<P_{2}$ when $\sigma^{x}<0$. In summary, this analysis shows that the correspondence between the zeros in the time-evolution of the order parameter and the occurrence of cusps in the 
return probability remains valid also in the presence of strong nearest-neighbor interactions.

In order to reduce spurious sign changes of $P_{1}-P_{2}$ due to finite-size effects in Figs. 20 and 19, we performed a moving average of $P_{1}, P_{2}$ over a small time window $0.2 \bar{\lambda}$.

\section{FINITE-SIZE EFFECTS}

In this last section, we discuss the relevance of the finite-size effects in the chaotic dynamical phase. For the sake of definiteness, we will focus on the model in Eq. (61).

The first observation in order is that the spin wave technique developed in Sec. III is rigorously valid in the thermodynamic limit, in which, as thoroughly discussed in Sec. II C, the collective spin can be treated as a classical degree of freedom. For the LMG model with $\tilde{J}_{k \neq 0}=0$, the spin-wave expansion (27) of the Hamiltonian allows one to compute the modifications to the classical evolution equations by accounting for the feedback from the quantum fluctuations of the $k=0$ mode in terms of $\Delta_{0}^{q q}, \Delta_{0}^{q p}, \Delta_{0}^{p p}$, which are suppressed as $N^{-1}$ [see Eq. (27)]. The presence of integrability-breaking perturbations $\tilde{J}_{k \neq 0} \neq 0$, as discussed in Sec. III, activates the quantum feedback from all the spin waves modes with $k \neq 0$, such as $\frac{1}{N s} \sum_{k \neq 0} \tilde{J}_{k} \Delta_{k}^{q q}(t)$ and similar terms in Eq. (50). In contrast to the feedback from the zeromode fluctuations, these latter terms have a finite limit as $N \rightarrow \infty$. For this reason, they have been properly taken into account in Sec. III and thereafter, while the feedback from the quantum fluctuations of the $k=0$ mode has been neglected throughout this paper.

In view of the above argument, all the results based on the time-dependent spin wave theory assume that the thermodynamic limit is taken at fixed time. The neglected finite-size effects typically set in at the (divergent) Ehrenfest time scale $T_{\mathrm{Eh}} \sim \mathcal{O}(\sqrt{N})$ discussed in Sec. II C. Thus, in Eqs. (50) and (54), as well as in all simulations reported in Sec. IV, the parameter $N$ plays the role of a discretization of the Brillouin zone integrals such as $\frac{1}{N s} \sum_{k \neq 0} \tilde{J}_{k} \Delta_{k}^{q q}(t) \sim \int_{-\pi}^{\pi} \frac{d k}{2 \pi s} \tilde{J}_{k} \Delta_{k}^{q q}(t)$, rather than properly accounting for actual finite-size effects.

The spin wave analysis carried out in the previous sections predicts the occurrence of a chaotic dynamical phase in perturbed mean-field models in the thermodynamic limit $N \rightarrow \infty$, which corresponds to a transient paramagnetic evolution followed by localization of the collective spin within one of the two ferromagnetic wells. It is then important to estimate how large $N$ should be in practice for this phenomenon to occur. In order to do this, we resort both to semiclassical arguments (cf. Sec. II C) as well as to MPS-TDVP simulations (cf. Sec. V). As explained in Sec. II C, within the lowest-order semiclassical expansion or TWA ${ }^{77}$, the quantum corrections to the classical motion amount to the replacement of the classical trajectory with the classical (Liouville) evolution of a Gaussian wavepacket in phase space cen- tered around an initial condition $\theta_{0}, \phi_{0}$. The width of this distribution is given by the quantum uncertainty of the transverse components $\tilde{\sigma}_{k=0}^{X} / N, \tilde{\sigma}_{k=0}^{Y} / N$ of the rescaled collective spin, which amount to $\sqrt{\tilde{q}_{0}^{2}} / \sqrt{N s}$ and $\sqrt{\tilde{p}_{0}^{2}} / \sqrt{N s}$, respectively [see Eq. (26)], and hence are proportional to $1 / \sqrt{N}$. The result of this approximation is visualized in Fig. 21, where the asymptotic magnetization is shown for quenches to $g / \bar{\lambda}=1.03, J / \bar{\lambda}=0.25$ as a function of the initial condition $\left(\phi_{0}, \cos \theta_{0}\right)$, with the same color conventions as in Fig. 7: The dynamical order parameter for a system of finite size $N$ corresponds to replacing the asymptotic magnetization of each point in this non-equilibrium phase diagram with its average over a Gaussian distribution centered at $\left(\phi_{0}=0, \cos \theta_{0}=0\right)$ with width of order $1 / \sqrt{N}$, pictorially represented by the circles in Fig. 21, corresponding to various values of $N$. This width can be viewed as a phase space coarsegraining scale in the presence of a system with finite size $N$. We see that within the chaotic dynamical phase, for small $N$, the wavepacket encompasses several initial conditions $\theta_{0}, \phi_{0}$ whose evolutions end up into distinct ferromagnetic sectors. Accordingly, after a transient, the actual many-body wavefunction is expected to realize a quantum superposition of two wavepackets localized in the two distinct ferromagnetic sectors, i.e., a so-called cat state. Hence, for sufficiently small $N$, this quantum superposition is expected to blur the critical region $\mathrm{C}$ in the diagram. A classical-like behavior characterized by a non-vanishing average magnetization is expected to be seen only when the size of the initial wavepacket becomes smaller than the distance between the phase space boundaries of regions with a definite sign of the asymptotic magnetization, which happens for sufficiently large $N$. Estimates based on the spin wave approximation in Sec. IV and in particular on the size of the largest spots of region $\mathrm{C}$ in Fig. 7 and in analogous diagrams for a range of parameters suggest that the minimal system size $N$ required in order to observe localization of the wavepacket within a single sector should lie in the range $10^{2} \div 10^{3}$. This agrees with the observed convergence of the MPSTDVP simulations within this region upon raising the bond dimension, as shown in Sec. V and in Ref. 66.

For smaller $N$, the remnant of the chaotic dynamical phase is expected to be the formation of cat states, which can in principle be detected by inspecting the evolution of the full statistics of the order parameter, rather than only of its the average. This study can actually be carried out via exact diagonalization of the Hamiltonian for a system size $N$ up to 16 . In this regime, however, finite-size effects are predominant (cf. Fig. 6). This can be easily understood within the semiclassical picture illustrated in Fig. 21, by observing that the order of magnitude $\mathcal{O}(1 / \sqrt{N})$ of the width of a spin-coherent wavepacket is comparable with the global width $\mathcal{O}(1)$ of the whole phase space when $N=16$, whereby the evolution results in a complicated superposition and interference of ferromagnetic, paramagnetic and "chaotic" 


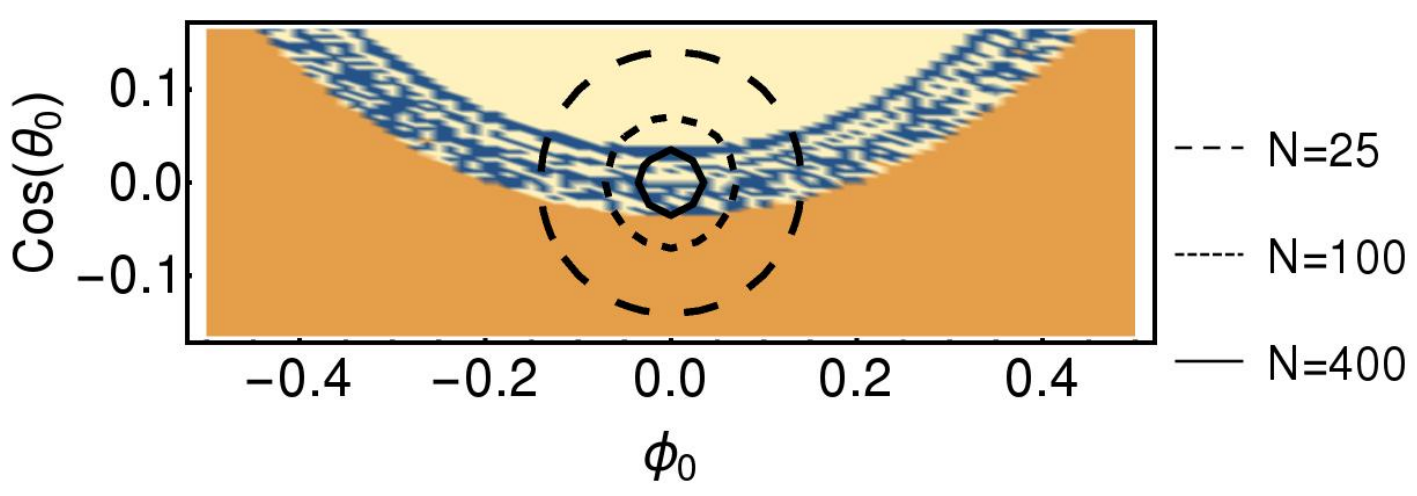

Figure 21. [Color online] Non-equilibrium phase diagram for quenches with fixed post-quench parameters $g / \bar{\lambda}=1.03$, $J / \bar{\lambda}=0.25$ as a function of the direction on the Bloch sphere of the pre-quench fully polarized spin-coherent initial state, parameterized by the canonically conjugated phase space coordinates $\phi_{0}$ and $\cos \theta_{0}$ (cf. Sec. III C). As in Fig. 7, this plot is obtained via numerically integrating the evolution equations of the time-dependent spin wave theory in the thermodynamic limit, with the same graphical conventions thereof. The lowest-order finite-size correction consists in replacing a classical, uncertainty-free initial condition, specified by $\left(\phi_{0}, \cos \theta_{0}\right)$, with a Gaussian wavepacket in phase space centered around it, with linear extension $\sqrt{\hbar_{\mathrm{eff}}}=1 / \sqrt{N s}$, which takes into account the quantum uncertainty at the lowest order in the semiclassical expansion, as discussed in Sec. II C. The circles superimposed to the diagram indicate the width of these Gaussian distributions centered around $(0,0)$ for various values of $N$, corresponding to quenches from a ground state of the pre-quench Hamiltonian with $g_{0}=0$ considered in all simulations reported in this work. We see that for $N \lesssim 10^{2}$ the corresponding wavepacket encompasses initial conditions eventually belonging to all possible phases of the model. Accordingly, one expects the chaotic dynamical phase to be blurred by these quantum fluctuations when $N$ is sufficiently small. This effect is more severe when $N$ is in the range $\lesssim 16$ accessible to full exact diagonalization of the Hamiltonian (61), which makes it hard to observe signatures of the chaotic dynamical phase via this exact method. The latter is observed in MPS-TDVP simulations with $N$ in the range $10^{2} \div 10^{3}$ and stronger perturbation $J / \bar{\lambda} \approx 0.5$, as reported in Sec. V, in correspondence of which the extension of the regions with a uniform sign of the asymptotic magnetization becomes sufficiently large compared to the coarse-graining scale $1 / \sqrt{N}$.

classical trajectories. For this reason we do not report the relative results here, and we leave a detailed investigation of this issue via finer numerical techniques to future studies.

We emphasize, however, that by tuning continuously the parameters across a phase boundary one should observe cat states for arbitrarily large $N$ : in fact, the evolution governed by a finite matrix has to depend smoothly on the parameters, and the time-evolved wavefunction cannot undergo "discontinuous" transitions at finite $N$, as is well-known from general theory. However, by the above semiclassical arguments, such cat states are expected to be confined within thin "layers" around the phase boundaries, whose width should shrink upon increasing $N$.

We conclude with two remarks on the crossover between quantum-mechanical behavior and its classical limit in the phenomena presented in this work as well as in Ref. 66. First, we note that the scale $1 / \sqrt{N}$, which is associated with the extension of the Wigner function of spin-coherent states, clearly represents the characteristic distance in phase space beyond which two spin-coherent states become effectively orthogonal in Hilbert space. In fact, two initial spin-coherent configurations separated by a smaller distance in phase space cannot localize in two distinct sectors after a certain time $t$, because they have non-vanishing initial overlap and hence cannot become orthogonal at any time due to unitarity of quantum time- evolution. Accordingly, the "collective chaotic behavior" unveiled in this work can arise in this quantum system only when the phase space coarse-graining scale $1 / \sqrt{N}$ provides a sufficiently fine resolution for resolving the different classical outcomes. There is no basic contradiction in the emergence of a classical-like chaos in a quantum system, as long as two nearby initial conditions, whose classical evolution undergoes exponentially fast separation, correspond to orthogonal initial vectors in Hilbert space. In this work we have shown that this can occur in systems with competition of long- and short-range interactions driven close to a dynamical phase transition, provided the system size $N$ is sufficiently large.

The second, related, remark is that, although the emergence of a classical-like collective chaotic behavior involves a subtle interplay between the thermodynamic limit and the time evolution, in practice there is no sharp distinction between cat states and classical sensitivity of the asymptotic magnetization with respect to the parameters of the system. In fact, in both cases, experimental measurements of the collective magnetization will result in a distribution characterized by two peaks, and understanding whether the origin of such a macroscopic superposition is quantum-coherent (as in a cat state) or classical-incoherent (as would result from unavoidable experimental errors) would actually be unfeasible, due to fast decoherence of the cat state (as for the original Schrödinger's cat!). 


\section{CONCLUSIONS}

This paper has been devoted to the analysis of the impact of non-equilibrium fluctuations on mean-field dynamical phase transitions. We have considered as unperturbed Hamiltonian a spin model with all-to-all couplings (2), whose dynamics in the thermodynamic limit are equivalent to the classical evolution of the collective spin orientation (see Sec. II). This has allowed us analyse the effect of any integrability-breaking interaction in terms of a systematic spin-wave expansion (see Sec. III). Through this time-dependent spin wave theory we have found a general phenomenon concerning perturbations of dynamical critical points: fluctuations dominate the dynamics and act as a self-generated quantum friction, which makes the order parameter eventually remain trapped in one of them in a pseudo-random fashion. We refer to this phase as chaotic, since the asymptotic sign of the order parameter depends with extreme sensitivity on the initial conditions and on the Hamiltonian parameters (see Secs. IV A and IV B). The existence of this peculiar dynamical behavior has been benchmarked with numerical methods based on a timedependent variational principle developed on the matrix product state manifold, and shown to persist even for stronger integrability-breaking couplings (see Sec. V). We have also studied the signatures of this novel dynamical phase on the space-time dependent correlation functions (see Sec. IV C), as well as demonstrated that for sufficiently slow ramps of the transverse magnetic field the chaotic phase gradually fades away (see Sec. IVD).

A straightforward and interesting extension of our analysis would consist in considering the sudden quench of integrability-breaking terms in the Dicke model, describing the interaction of several two-level atoms (spins) with a collective cavity photonic mode. The Dicke model possesses a rich dynamical phase diagram resulting from a quantum quench of the light-matter coupling ${ }^{42,92-94}$. The potential onset of a similar chaotic dynamical phase, monitored by photonic observables directly accessible in cavity quantum-electrodynamics experiments, could represent a welcome experimental verification of the phenomena discussed in this work.

Finally, it would be interesting to inspect the effect on mean-field dynamical critical phases of a weak spatial disorder, which can be accounted for in the timedependent spin wave treatment: a natural, intriguing question would be to establish whether the competition of quantum fluctuations and classical spatial inhomogeneities would enhance or suppress the novel nonequilibrium phase discussed in this paper.

Acknowledgments. We thank E. Demler, F. Essler, Y. Gefen, A. Rosch for useful discussions. J. M. acknowledges support from the Alexander Von Humboldt foundation. J. M. is supported by the European Union's Horizon 2020 research and innovation programme under the Marie Sklodowska-Curie grant agreement No 745608 (QUAKE4PRELIMAT). B. Z. is supported by the Advanced grant of European Research Council (ERC), No. 694544 - OMNES
1 A. Polkovnikov, K. Sengupta, A. Silva, and M. Vengalattore, Rev. Mod. Phys. 83, 863 (2011).

2 A. Lamacraft and J. Moore, in Ultracold Bosonic and Fermionic Gases, edited by K. Levin, A. Fetter, and D. Stamper-Kurn (Elsevier, Amsterdam, 2012) Chap. 7.

3 C. Gogolin and J. Eisert, Rep. Prog. Phys. 79, 056001 (2016).

4 J. Berges, S. Borsányi, and C. Wetterich, Phys. Rev. Lett. 93, 142002 (2004).

5 J. Berges, AIP Conf. Proc. 739, 3 (2004).

6 C. Kollath, A. M. Läuchli, and E. Altman, Phys. Rev. Lett. 98, 180601 (2007).

7 M. Moeckel and S. Kehrein, Phys. Rev. Lett. 100, 175702 (2008).

8 A. Rosch, D. Rasch, B. Binz, and M. Vojta, Phys. Rev. Lett. 101, 265301 (2008).

9 M. Moeckel and S. Kehrein, Ann. Phys. 324, 2146 (2009).

10 M. Gring, M. Kuhnert, T. Langen, T. Kitagawa, B. Rauer, M. Schreitl, I. Mazets, D. A. Smith, E. Demler, and J. Schmiedmayer, Science 337, 1318 (2012).

11 J. Marino and A. Silva, Phys. Rev. B 89, 024303 (2014).

12 T. Langen, R. Geiger, M. Kuhnert, B. Rauer, and J. Schmiedmayer, Nat. Phys. 9, 640 (2013).

13 N. Nessi, A. Iucci, and M. A. Cazalilla, Phys. Rev. Lett. 113, 210402 (2014).

14 T. Langen, T. Gasenzer, and J. Schmiedmayer, J. Stat.
Mech. 064009 (2016).

15 T. Kinoshita, T. Wenger, and D. S. Weiss, Nature 440, 900 (2006).

16 M. A. Cazalilla, Phys. Rev. Lett. 97, 156403 (2006).

17 M. Rigol, V. Dunjko, V. Yurovsky, and M. Olshanii, Phys. Rev. Lett. 98, 050405 (2007).

18 M. Cramer, C. M. Dawson, J. Eisert, and T. J. Osborne, Phys. Rev. Lett. 100, 030602 (2008).

19 T. Barthel and U. Schollwöck, Phys. Rev. Lett. 100, 100601 (2008).

20 D. Fioretto and G. Mussardo, New J. Phys. 12, 055015 (2010).

21 J.-S. Caux and R. M. Konik, Phys. Rev. Lett. 109, 175301 (2012).

22 P. Calabrese, F. H. L. Essler, and M. Fagotti, Phys. Rev. Lett. 106, 227203 (2011).

23 T. Langen, S. Erne, R. Geiger, B. Rauer, T. Schweigler, M. Kuhnert, W. Rohringer, I. Mazets, T. Gasenzer, and J. Schmiedmayer, Science 348, 207 (2015).

${ }^{24}$ F. H. L. Essler and M. Fagotti, J. Stat. Mech. 064002 (2016).

25 P. Calabrese and J. Cardy, J. Stat. Mech. P06008 (2007).

26 M. Kollar, F. A. Wolf, and M. Eckstein, Phys. Rev. B 84, 054304 (2011).

27 M. Stark and M. Kollar, arXiv:1308.1610 (2013).

${ }^{28}$ F. H. L. Essler, S. Kehrein, S. R. Manmana, and N. J. 
Robinson, Phys. Rev. B 89, 165104 (2014).

29 B. Bertini, F. H. L. Essler, S. Groha, and N. J. Robinson, Phys. Rev. Lett. 115, 180601 (2015).

30 B. Bertini, F. H. L. Essler, S. Groha, and N. J. Robinson, Phys. Rev. B 94, 245117 (2016).

31 M. Marcuzzi, J. Marino, A. Gambassi, and A. Silva, Phys. Rev. Lett. 111, 197203 (2013).

32 M. Babadi, E. Demler, and M. Knap, Phys. Rev. X 5, 041005 (2015).

33 M. Marcuzzi, J. Marino, A. Gambassi, and A. Silva, Phys. Rev. B 94, 214304 (2016).

${ }^{34}$ I. Frérot, P. Naldesi, and T. Roscilde, Phys. Rev. Lett. 120, 050401 (2018).

35 R. A. Barankov, L. S. Levitov, and B. Z. Spivak, Phys. Rev. Lett. 93, 160401 (2004).

36 R. A. Barankov and L. S. Levitov, Phys. Rev. Lett. 96, 230403 (2006).

37 E. A. Yuzbashyan, O. Tsyplyatyev, and B. L. Altshuler, Phys. Rev. Lett. 96, 097005 (2006).

38 V. Gurarie, Phys. Rev. Lett. 103, 075301 (2009).

39 M. Eckstein, M. Kollar, and P. Werner, Phys. Rev. Lett. 103, 056403 (2009).

40 M. Schiró and M. Fabrizio, Phys. Rev. Lett. 105, 076401 (2010).

41 B. Sciolla and G. Biroli, Phys. Rev. Lett. 105, 220401 (2010).

42 B. Sciolla and G. Biroli, J. Stat. Mech. P11003 (2011).

43 A. Gambassi and P. Calabrese, Europhys. Lett. 95, 66007 (2011).

44 M. Schiró and M. Fabrizio, Phys. Rev. B 83, 165105 (2011).

45 M. Sandri, M. Schirò, and M. Fabrizio, Phys. Rev. B. 86, 075122 (2012).

46 B. Sciolla and G. Biroli, Phys. Rev. B 88, 201110 (2013).

47 M. S. Foster, M. Dzero, V. Gurarie, and E. A. Yuzbashyan, Phys. Rev. B 88, 104511 (2013).

48 M. S. Foster, V. Gurarie, M. Dzero, and E. A. Yuzbashyan, Phys. Rev. Lett. 113, 076403 (2014).

49 P. Smacchia, M. Knap, E. Demler, and A. Silva, Phys. Rev. B 91, 205136 (2015).

${ }^{50}$ F. Peronaci, M. Schiró, and M. Capone, Phys. Rev. Lett. 115, 257001 (2015).

51 E. A. Yuzbashyan, M. Dzero, V. Gurarie, and M. S. Foster, Phys. Rev. A 91, 033628 (2015).

52 J. C. Halimeh, V. Zauner-Stauber, I. P. McCulloch, I. de Vega, U. Schollwöck, and M. Kastner, Phys. Rev. B 95, 024302 (2017).

${ }^{53}$ I. Homrighausen, N. O. Abeling, V. Zauner-Stauber, and J. C. Halimeh, Phys. Rev. B 96, 104436 (2017).

${ }^{54}$ H.-K. Janssen, B. Schaub, and B. Schmittmann, Z. Phys. B Cond. Mat. 73, 539 (1989).

55 P. Calabrese and A. Gambassi, J. Phys. A: Math. Gen. 38, R133 (2005).

56 A. Chandran, A. Nanduri, S. S. Gubser, and S. L. Sondhi, Phys. Rev. B 88, 024306 (2013).

57 A. Maraga, A. Chiocchetta, A. Mitra, and A. Gambassi, Phys. Rev. E 92, 042151 (2015).

58 A. Chiocchetta, M. Tavora, A. Gambassi, and A. Mitra, Phys. Rev. B 91, 220302 (2015).

59 A. Chiocchetta, A. Gambassi, S. Diehl, and J. Marino, Phys. Rev. Lett. 118, 135701 (2017).

60 J. Zhang, G. Pagano, P. W. Hess, A. Kyprianidis, P. Becker, H. Kaplan, A. V. Gorshkov, Z. X. Gong, and C. Monroe, Nature 551, 601 (2017).

${ }^{61}$ M. Heyl, A. Polkovnikov, and S. Kehrein, Phys. Rev. Lett.
110, 135704 (2013).

${ }^{62}$ P. Jurcevic, H. Shen, P. Hauke, C. Maier, T. Brydges, C. Hempel, B. P. Lanyon, M. Heyl, R. Blatt, and C. F. Roos, Phys. Rev. Lett. 119, 080501 (2017).

63 B. Žunkovič, M. Heyl, M. Knap, and A. Silva, Phys. Rev. Lett. 120, 130601 (2018).

64 S. A. Weidinger, M. Heyl, A. Silva, and M. Knap, Phys. Rev. B 96, 134313 (2017).

65 J. Lang, B. Frank, and J. C. Halimeh, Phys. Rev. Lett. 121, 130603 (2018).

66 A. Lerose, J. Marino, B. Žunkovič, A. Gambassi, and A. Silva, Phys. Rev. Lett. 120, 130603 (2018).

67 J. Strzalko, J. Grabski, A. Stefanski, P. Perlikowski, and T. Kapitaniak, The Mathematical Intelligencer, 32, 54 (2010).

68 S. Sachdev, Quantum Phase Transitions (Cambridge University Press, 2011).

69 P. Calabrese, F. H. L. Essler, and M. Fagotti, J. Stat. Mech. P07016 (2012).

70 H. Lipkin, N. Meshkov, and A. Glick, Nucl. Phys. 62, 188 (1965).

71 A. Das, K. Sengupta, D. Sen, and B. K. Chakrabarti, Phys. Rev. B 74, 144423 (2006).

72 B. Žunkovič, A. Silva, and M. Fabrizio, Phil. Trans. R. Soc. A 374, 20150160 (2016).

73 L. D. Landau and E. M. Lifsits, Mechanics (ButterworthHeinemann, 1976).

74 The LMG model is solvable by Bethe-ansatz for all $N$ (see Ref. 95), which allows in principle to compute analytically the ground state as well as the non-equilibrium properties. However, this exact solution in quite unpractical for large $N$, and a semiclassical approach turns out to be simpler and more powerful in order to understand the behavior of the system.

75 R. G. Littlejohn, Phys. Rep. 138, 193 (1986).

76 M. Brack and R. K. Bhaduri, Semiclassical Physics (Addison-Wesley, 1997).

77 A. Polkovnikov, Ann. Phys. 325, 1790 (2010).

78 This fact is crucial for the phenomenology of the chaotic dynamical ferromagnetic phase, see Sec. IV.

79 T. Mori, Phys. Rev. E 96, 012134 (2017).

${ }^{80}$ G. H. Wannier, Statistical Physics (Dover, 1966).

81 E. H. Lieb, J. P. Solovej, R. Seiringer, and J. Yngvason, The Mathematics of the Bose Gas and its Condensation (Birkhäuser Basel, 2005).

82 The ordering of the operators $Q, P$ is actually immaterial, as differently ordered expressions differ by terms of higher order in $1 / N$, suppressed in the thermodynamic limit. Indeed, as explained in Sec. II, when $N \rightarrow \infty$ the behavior of the collective mode is classical.

83 Note, however, that completely different phenomena are expected in the presence of antiferromagnetic long-range interactions, i.e., when $\lambda<0$ : see, e.g., Ref. 96.

${ }^{84}$ P. Calabrese and J. Cardy, Phys. Rev. Lett. 96, 136801 (2006).

85 E. H. Lieb and D. W. Robinson, Commun. Math. Phys. 28, 251 (1972).

86 A. Russomanno, A. Silva, and G. E. Santoro, Phys. Rev. Lett. 109, 257201 (2012).

87 A. Lazarides, A. Das, and R. Moessner, Phys. Rev. Lett. 112, 150401 (2014).

88 M. Bukov, L. D'Alessio, and A. Polkovnikov, Adv. Phys. 64, 2 (2015).

89 J. Haegeman, C. Lubich, I. Oseledets, B. Vandereycken, 
and F. Verstraete, Phys. Rev. B 94, 165116 (2016).

90 C. Lubich, I. V. Oseledets, and B. Vandereycken, SIAM J. Numer. Anal 53, 917 (2015).

91 M. Heyl, Phys. Rev. Lett. 113, 205701 (2014).

92 C. Emary and T. Brandes, Phys. Rev. E 67, 066203 (2003).

93 J. Keeling, M. J. Bhaseen, and B. D. Simons, Phys. Rev.
Lett. 105, 043001 (2010).

${ }^{94}$ M. J. Bhaseen, J. Mayoh, B. D. Simons, and J. Keeling, Phys. Rev. A 85, 013817 (2012).

${ }^{95}$ G. Ortiz, R. Somma, J. Dukelsky, and S. Rombouts, Nucl. Phys. B 707, 421 (2005).

96 A. Giuliani, J. L. Lebowitz, and E. H. Lieb, Phys. Rev. B 74, 064420 (2006). 